\title{
Nanomaterials as Photothermal Agents for Biomedical Applications
}

\author{
Antonia Cuello, ${ }^{1}$ Silvestre Bongiovanni Abel, ${ }^{2}$ César A. Barbero, ${ }^{1}$ Inés Yslas, ${ }^{1}$ and \\ María Molina ${ }^{1 *}$ \\ ${ }^{1}$ Instituto de Investigaciones en Tecnologías Energéticas y Materiales Avanzados (IITEMA), Fac. Ciencias Exactas Fisicoquímicas y \\ Naturales, Universidad Nacional de Río Cuarto (UNRC)-Consejo Nacional de Investigaciones Científicas y Técnicas (CONICET), Río \\ Cuarto, Argentina. \\ ${ }^{2}$ Instituto de Investigaciones en Ciencia y Tecnología de Materiales (INTEMA), Universidad Nacional de Mar del Plata (UNMdP)- \\ Consejo Nacional de Investigaciones Científicas y Técnicas (CONICET), Mar del Plata, Argentina. \\ ${ }^{*}$ Corresponding author. E-mail: mamolina7@gmail.com
}

\begin{abstract}
Photothermal therapy (PTT) is a potentially curative treatment modality that in recent years has been the object of growing interest and rapid technological advances due to its specific therapeutic efficacy and because it is a non-invasive technique. Nowadays, several nanomaterials have been developed as photothermal agents including metallic and carbon-based nanoparticles, conducting polymers, and different kinds of nanocomposites, among others. In this article, the most relevant applications of these photothermal nano-agents in antibacterial and anticancer therapy are reviewed.
\end{abstract}

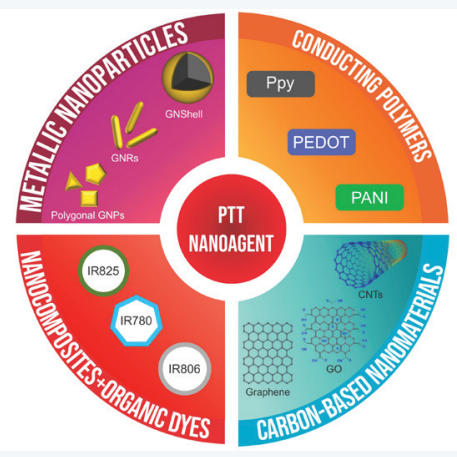

\section{Keywords:}

photothermal nanoagents, PTT, antibacterial therapy, anticancer therapy

\section{Introduction}

Photothermal therapy (PTT) is an alternative treatment to conventional therapies that lately has been the object of increasing interest in the scientific community. This is due to its specific therapeutic efficacy, since it is a non-invasive technique and has the potential for combination in the same approach diagnosis and therapy (Millenbaugh et al. 2015). PTT is based on the use of photothermal agents that absorb radiation in the near infrared (NIR) region and convert the absorbed energy into heat through a non-radiative mechanism without oxygen consumption. Therefore, PTT requires the use of nanomaterials that exhibit high absorption in the NIR region of the electromagnetic spectrum. NIR absorbing nanomaterials are promising agents for PTT, allowing application deep in the tissue. Human tissue has almost no absorption of NIR radiation, while other body constituents, such as hemoglobin and water, have minimal absorption (Weissleder 2001). The advantage of using nanomaterials is that the light absorbed can be rapidly converted into heat, greatly increasing the temperature of the irradiated zone, which heats up the whole organ, avoiding the drawback of macroscopic hyperthermia (Zhou et al. 2018). The amount of heat induced by NIR irradiation is related to different parameters, namely, NIR light intensity, irradiation time, and the concentration of the photothermally active nanomaterials. Nanoparticles under irradiation scatter some of the photons and absorb the others. The energy produced by the absorbed photons can be released in two ways - photon emission or phonon emission. The emission of phonons generates heat. To obtain a good candidate for PTT therapies, nanoparticles need to show large absorption efficiencies and low luminescence quantum yields. This is what will ensure a large photothermal conversion efficiency. 
It was reported that temperatures greater than $41^{\circ} \mathrm{C}$ inhibit cell proliferation and mobility, which, in turn, can increase permeability, trigger cell wall damage and increase cellular lysis (Tsuchido et al. 1985). Intracellular temperature causes irreversible cellular damage, such as denaturation of cell proteins/enzymes, and, therefore, interferes in essential intracellular reactions, leading to the death of cells in a rapid and effective manner. In addition, damage to the DNA structure is triggered, causing genotoxic effects that include breakage of the DNA strand and disruption of the chromosomal cell membrane (Jia et al. 2017, Korupalli et al. 2017, Wu et al. 2013). The heat generated by NIR exposition also could break the cell membrane. Additionally, some researchers even predict NIR-light-triggered therapy platforms to be an innovative and exciting possibility for clinical nanomedicine applications.

This review focuses on the latest progress and future development trends in the applications of nanomaterials as photothermal agents for biological applications, with special emphasis in antibacterial and anticancer therapy (Figure 1). We review the use of nanomaterials as photothermal absorbers, including metallic and carbon-based nanoparticles, nanocomposites and conductive polymers.

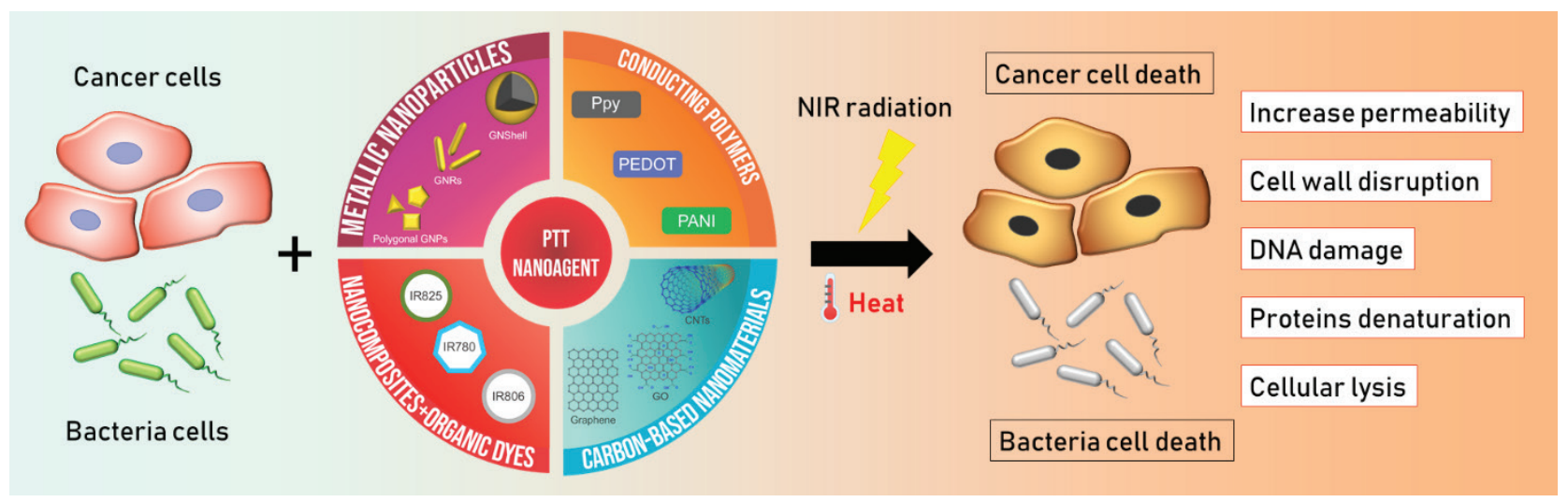

Figure 1. Different nanomaterials as photothermal agents for biological applications.

\section{Progress on utilizing tremendous potential PTT for mitigating bacterial infections}

Infectious diseases caused by bacteria are a problem that has potentially catastrophic global consequences related to health. In recent decades, the emergence of bacteria resistant to multiple antibiotics has become a common cause of infectious diseases due to overprescribing and indiscriminate use of antibiotics (World Health Organization 2014). The proportion of antibiotics-resistant bacterial isolates has been alarmingly increasing through the intrinsic and acquired mechanisms of antimicrobial resistance (Andersson et al. 2010). The problem of antibiotic-resistant bacteria and the high costs of medical care encourages researchers to come up with innovative approaches to develop more effective antibacterial agents that overcome bacterial resistance and reduce costs. Thus, there is an urgent need to develop novel antibacterial agents and therapies to combat pathogens in a safer and more effective way (Zharov et al. 2006).

It is well known that most pathogenic bacteria die at temperatures above $40^{\circ} \mathrm{C}$ (Mackowiak 1981). Therefore, the PTT approach has been widely applied as bacteria-killing mechanism for the last few years. Recently, He et al. (He et al. 2018) designed an antimicrobial hybrid based on polydopamine (PDA)-coated gold nanorods (PDA@GNRs) grafted with glycol chitosan (GCS-PDA@GNRs) and loaded with daptomycin (DAP-GCS-PDA@GNRs) which improves antibacterial activity and reduces side effects in vivo. This hybrid material showed an outstanding chemo-photothermal synergistic therapeutic effect on the abscess, leading to sufficient bacteria eradication that could thoroughly ablate abscess while reducing the damage to normal tissue, accelerating wound healing. Khan et al. reported the synergistic biocide effect of graphene oxide (GO) and Nd-YAG laser for antibacterial and antifungal treatments (Shahnawaz Khan et al. 2015). The authors suggest that this will serve as a novel and promising therapeutic strategy and has great potential to serve for PTT against pathogenic microorganisms. It was also reported that GO offered high efficiency for photothermal treatment (Pereyra et al. 2018, Wu et al. 2013). The combination of PTT with pharmacotherapy can not only significantly improve the antibacterial efficiency towards drug-resistant bacteria, but also readily reduce antibiotic dosage, thus providing a potential solution to the problems of drug-resistant bacteria.

Other authors have shown that during the treatment with a PTT agent, as a result of a thermal shock, an excess of reactive oxygen species (ROS) could be produced. Thus, ROS can cause protein or lipids damage leading to cell death. ROS are generally well defined as containing oxygen and active chemical substances, and usually are classified as one of two 
kinds: free radicals and nonradicals (Birben et al. 2012). ROS that are commonly observed in biological systems include hydroxyl radicals, superoxide, and nitric oxide, ozone, hydroxide, peroxynitrite, and hydrogen peroxide.

Bacteria that adhere to surfaces of materials or tissues have the tendency to encase themselves in a hydrated matrix, forming a layer known as biofilm that is made of a bacterial community embedded in a self-produced matrix of extracellular polymeric substances (EPS) (Singh et al. 2017). More than $80 \%$ of human infectious diseases are related to the formation of biofilms. Bacterial biofilms act as a barrier to prevent the penetration of antibiotics in bacterial cells; therefore, it is generally not effective to destroy such biofilms, which can also generate resistance to antimicrobial agents and result in antibiotic treatment failure. Other scientists have applied PTT to disrupt the biofilm structure by physical effects of the heat that is generated by light absorption of materials without giving rise to drug-resistance of pathogenic bacteria (Tan et al. 2018, Zhao et al. 2017). Therefore, water may be eventually removed from the biofilm causing dehydration and as consequence, the death of the pathogens, triggering denaturation of the cell wall and protein/enzymes, leading to the leakage of cellular content and bacterial death. (Figure 2).
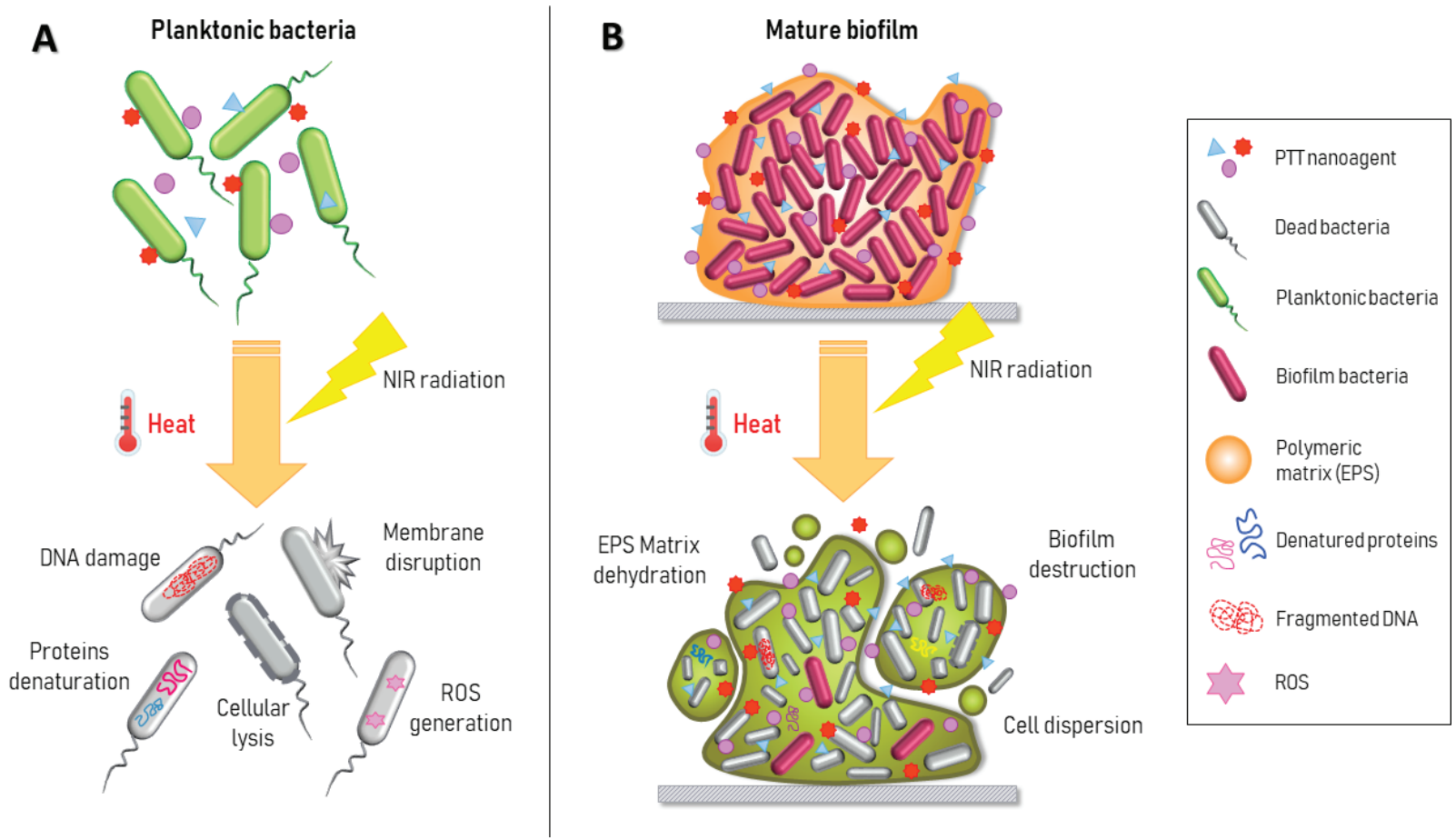

Figure 2. Schematic representation of the possible mechanism of nanomaterials in combination with NIR irradiation for effectively killing planktonic bacteria (A) and for destroying bacterial biofilms (B) using PTT.

\section{PTT for anticancer therapy}

Cancer is one of the leading causes of death worldwide, and traditional therapies have enormous drawbacks including side effects and the development of resistance of the cells to the conventional drugs (Housman et al. 2014). That is why PTT is an attractive alternative anticancer therapy, since it can provoke cell death via protein denaturation and the subsequent tumor regression with minimal invasion and almost no side effects (Chen et al. 2018).

However, to treat tumors that are not at the surface, the activating energy source needs to penetrate tissues, efficiently kill tumors and not damage healthy tissues. Thus, there is an urgent need of looking for nanoagents with high photothermal efficiency that can target tumor cells. In the last years, thermal therapies for treating cancer have received great attention using different heating sources such as laser, focused ultrasound, and microwaves. More interestingly, PTT using NIR induces minimal damage in both tumors and healthy tissues. Thus, there is an increasing interest in developing photothermal nanoagents capable to absorb NIR light and transduce it to heat in order to kill the malignant cells without damaging the surrounding healthy tissues (Wang et al. 2016). Several photoactive nanomaterials such as magnetic nanomaterials, carbon nanotubes, or gold nanoparticles, activated with NIR radiation, have proven to be effective in PTT treatment of various cancers (Cheng et al. 2014). Additionally, PTT in combination with other therapies, such as chemotherapy (Cao et al. 2017), or even other phototherapies, such as photodynamic therapy (PDT) (Liu et al. 2018, Wang et al. 2017), has gained great attention for increasing treatment efficacy. Furthermore, the combination of PTT with imaging is a new trend to develop theranostic systems for cancer therapy (Jung et al. 2018). 


\section{Nanomaterials as photothermal agents}

PTT has been introduced as a potent alternative therapeutic modality against drug-resistant bacterial infections, as well as against drug resistant cancer cells. So far, numerous photothermal nanoagents with strong NIR absorption have been explored for PTT, including gold nanorods, nanoshells, nanocages, nanostars, copper selenide nanoparticles, carbonous nanostructures (graphene oxide (GO), carbon nanotubes (CNTs)) and polymeric nanoparticles (Jaque et al. 2014). In fact, advances in nanomedicine have recently allowed us to manufacture multifunctional nanoparticles that offer diagnostic and therapeutic functions in a single environment (Menon et al. 2013). In PTT, within a few minutes upon NIR radiation, temperature rises locally due to the heat generation caused by the photothermal agents, which results in irreversible cell damage by hyperthermia effect. This hyperthermia process is triggered by applying pulses of light directly on the infected location, which causes tissue temperature to increase because it absorbs the energy of the light, releasing it as heat.

\subsection{Conducting polymers}

Conducting polymers exhibit conjugation in their molecular structure due to the presence of a $\pi$-electrons system delocalized over the whole polymeric main chain. Additionally, the presence of delocalized charges in the chains render them electrically conductive. However, NIR light absorption in conjugated polymers is related to the presence of low-energy $(<1.58 \mathrm{eV})$ electronic transitions. Therefore, all conjugated polymers, which can be conducting or semiconducting, could show NIR light absorption. The low cost, stability, biocompatibility, high biodegradability and versatility of conducting polymers allow using them on photothermal processes and therapy treatments. Under light irradiation, the polymer absorbs the energy and transforms it into localized heat that can be useful for generating a hyperthermia process in living systems (Chen et al. 2018, Pierini et al. 2018). Polyaniline (PANI), polypyrrole (PPy), and poly-(3,4-ethylenedioxythiophene) (PEDOT) are the most important conducting polymers studied for photothermal treatments. In this section, the most relevant and promising nanomaterials and composites based on these and other conducting polymers for PTT applications are described.

Taking into account the synthetic approach reported by Stejskal and coworkers (Stejskal et al. 2005), PANI nanoparticles stabilized with polyvinylpyrrolidone (PVP) as a hydrophilic agent were obtained by oxidative polymerization of the monomer salt. Spherical particles of c.a. $200 \mathrm{~nm}$ were obtained and fully characterized by several microscopic, light dispersion, and spectroscopic techniques. In vitro experiments revealed the successful incorporation of the nanoparticles into the cells, showing no cytotoxicity at high concentrations and unaltered morphology. The destruction of LM2 tumoral cells was achieved by combining the conducting nanoparticles with laser irradiation using NIR light at low power (Yslas et al. 2015). Furthermore, in vivo assays on an animal model were carried out using the same PANI nanoparticles. In this case, the tumors were injected with the nanomaterial and irradiated during 15 minutes with the same NIR light that was used for the in vitro experiments $\left(785 \mathrm{~nm}\right.$, power density of $\left.500 \mathrm{~mW} \mathrm{~cm}^{-2}\right)$. Results have shown that tumoral growth was inhibited after PTT with a significant reduction of tumor volume, suggesting that the efficient nanoparticles for PTT are a promising agent for anticancer clinical treatments (Ibarra et al. 2013). The same PANI nanoparticles generated by oxidative polymerization were also used to explore the antibacterial photothermal effect under Pseudomonas aeruginosa (P. aeruginosa), one of the most important pathogenic bacteria that causes nosocomial infections. The nanomaterials showed a synergistic effect between nanoparticles and NIR light (Bongiovanni Abel et al. 2018). In this sense, P. aeruginosa colony viability was reduced by more than $80 \%$ after irradiating for 15 minutes the bacteria exposed to the nanoparticles, demonstrating that the nanomaterial is an effective photothermal agent capable of inhibiting cellular growth for this bacteria type. The DNA fragmentation in the microorganism exposed to NIR light supports the mechanism of cell death. The authors revealed that the nanomaterial has a PTT effect similar or comparable to that of other materials (e.g. metal nanoparticles).

PANI nanoparticles stabilized with a thermosensitive polymer (PNIPAm) in combination with PVP were synthesized and the photothermal effect was evaluated (Bongiovanni Abel et al. 2014). To simulate the environment of the tumoral cell, where $\mathrm{pH}$ is lower and the temperature is higher than normal physiological conditions, assays were carried out in acidic media. After NIR laser irradiation, under similar conditions as those used by Ibarra et al. (Ibarra et al. 2013), a high transmittance decrease of the dispersion was observed in only 6 minutes, indicating the collapse of the PNIPAm stabilizer by the heating of the conducting polymer. Thermosensitive polymer chain aggregation was measured by turbidimetric techniques, resulting close to the $\operatorname{LCST}\left(32^{\circ} \mathrm{C}\right)$. The photothermal effect was dependent on nanoparticle concentration, reaching a temperature increment of $13{ }^{\circ} \mathrm{C}$ on PANI nanoparticles irradiated for 10 minutes. The thermosensitive properties of PANI/PNIPAm nanoparticles could be useful due to the formation, under heating, of polymeric aggregates that show a reversible behavior. Additionally, another novel method for obtaining PANI nanoparticles was recently reported by Bongiovanni Abel et al. (Bongiovanni Abel et al. 2018). Multifunctional PANI particles of controlled size and new properties, such as fluorescence, were obtained. First, the conducting polymer was chemically modified in order to add the fluorophore (dansyl chloride). After that, nanoparticles were formed using the solvent displacement method, which yielded monodisperse nanoparticles with a size of c.a. $104 \mathrm{~nm}$. The photothermal effect by application of NIR light was tested 
on nanoparticle dispersion, irradiating for 10 minutes. An increment of the local temperature was registered, reaching a total difference of $8^{\circ} \mathrm{C}$. The antibacterial PTT was also evaluated, inhibiting cellular growth in P. aeruginosa. However, in this case, the toxicity of the organic solvent (N-methylpyrrolidone) needs to be considered.

On the other hand, Calderón and coworkers obtained dendritic nanogels containing conducting polymers by semi-interpenetration (PNIPAm-dPG/PANI NGs) and studied their performance as in vivo PTT agent on tumoral cells (Molina et al. 2016). The nanoparticles exhibited diameters between 150-240 nm depending on the temperature of the media and showed spherical morphology. The resulting temperature increment depended on nanocomposite concentration and time of exposure under the irradiation. At higher concentrations $(80 \mu \mathrm{g} \mathrm{mL}-1)$, the temperature increase reached c.a. $20{ }^{\circ} \mathrm{C}$, demonstrating the efectiveness for their use as a potential photothermal agent. In vitro assays using A2780 cells revealed a markedly reduced viability upon NIR irradiation $(785 \mathrm{~nm})$. Additionally, in vivo experiments in mice with transplanted A2780 tumor showed a significant reduction of tumor volume under NIR laser at $500 \mathrm{~mW}$ power for 5 minutes. Evaluation at different times after injection of nanocomposites and irradiation allowed significant tumor growth inhibition, showing treatment efficacy.

As mentioned above, PPy is another one of the most used conducting polymers to generate a photothermal effect. Some works have shown the efficacy of this material for this application in living systems. For example, hollow PPy microspheres (PPyHMs) generated by one-step oil-water microemulsion method in an aqueous solution containing PVP was synthesized by Zha et al. (Zha et al. 2013). They have shown that it is possible to combine the photothermal activity of the conducting polymer with ultrasound imaging for evaluating the material as theranostic agent. Tumor ablation was successful, taking advantage of the strong absorption of PPy in the NIR therapeutic window. An increment of c.a. $30{ }^{\circ} \mathrm{C}$ was measured in a concentration of $150 \mu \mathrm{g} \mathrm{mL}^{-1}$ of the spherical dispersible particles (Figure 3). Moreover, in vivo ultrasound imaging facilitated the evaluation of PPy nanoparticles in a mouse model with tumor, showing excellent photothermal conversion efficiency by applying $0.64 \mathrm{~W} \mathrm{~cm}^{-2}$ laser power and easy identification of the particle location into the tumor. The same authors reported similar results with uniform PPy nanoparticles, where the stabilizer used was polyvinyl alcohol (PVA) and $\mathrm{FeCl}_{3}$ was employed as an oxidizing agent in the synthetic approach to obtain nanospheres with an average diameter of $\approx 46 \mathrm{~nm}$. Photothermal assays were made in a RPMI-1640 culture medium in the presence of conducting particles, and results revealed that temperature could be increased up to $34.5{ }^{\circ} \mathrm{C}$ after 10 minutes of NIR irradiation $(808 \mathrm{~nm}, 2 \mathrm{~W})$ for 10 minutes. A concentration-dependent effect was also observed in this case. In vitro studies confirmed good material photostability and biocompatibility, showing PTT ablation on HeLa cells (Zha et al. 2013). Wang et al. reported the application of PPy nanoparticles with a layer of natural red blood cell (RBC) on the surface for local heating generation. A comparison between PPy NPs, PPy-PEG NPs, and PPy@RBC NPs was made in order to find strategies that allow minimizing the dosage of nanoparticles into the living system. The three kinds of nanoparticles were tested as PTT agents on HCT116 cells at concentrations between $0-40 \mu \mathrm{g} \mathrm{mL}^{-1}$ by applying NIR light with power density between $0.75-1.50 \mathrm{~W} \mathrm{~cm}^{-2}$ for 3 minutes, repeating three cycles in each case. A three-factor dependence was found in the hyperthermia treatment: concentration, exposure time, and light power. The best performance was observed with PPy@ RBC NPs: $53.2{ }^{\circ} \mathrm{C}$ increment in temperature at $40 \mu \mathrm{g} \mathrm{mL}^{-1}, 1.50 \mathrm{~W} \mathrm{~cm}^{-2}$, and $180 \mathrm{~seconds}$ of laser irradiation, showing, photothermal conversion efficiency close to $40 \%$. Additionally, nude male mice were used to try the in vivo PTT, concluding that a low-dose of PPy@RBC NPs can be sufficient to observe tumor ablation (Wang et al. 2017). 


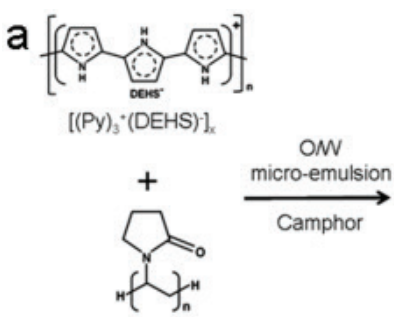

PVP
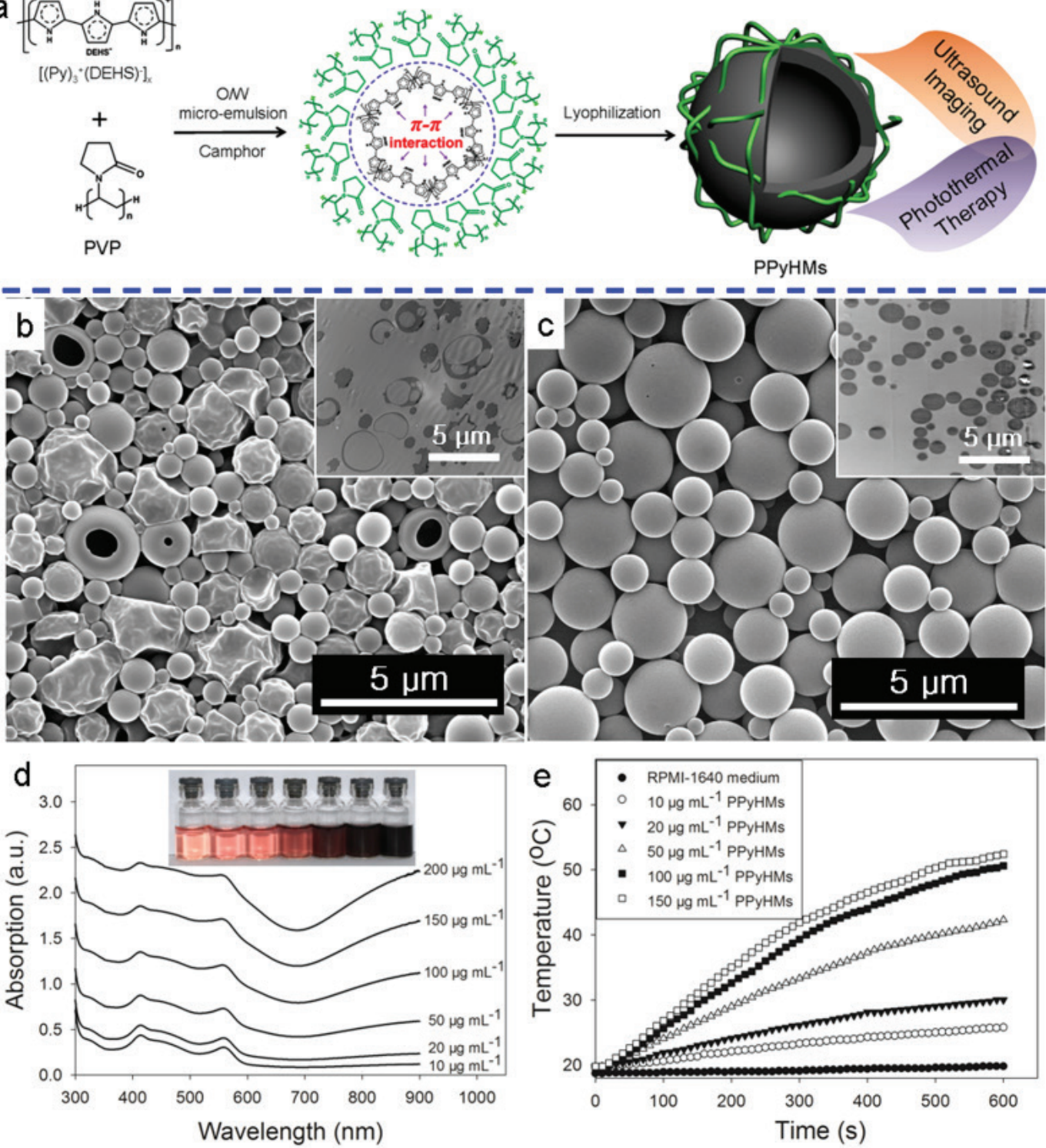

Figure 3. Preparation and characterization of water-dispersible PPyHMs. (a) Schematic illustration of echogenic PPyHMs formation for combined US imaging and PTT via a facile O/W microemulsion method; SEM and ultrathin-section TEM images of obtained PPyHMs with (b) PVP and (c) PVA as stabilizers; (d) UV-vis-NIR spectra of various concentrations PPyHMs dispersed in RPMI-1640 culture medium, inset was the photograph for various concentrations of PPyHMs dispersed in RPMI-1640 culture medium, indicating good dispersibility; (e) Heating curves of PPyHMs in RPMI-1640 culture medium at different concentrations under $808 \mathrm{~nm}$ laser irradiation. Reproduced with permission from reference (Zha et al. 2013) which is licensed under a Creative Commons Attribution-NonCommercial-NoDerivs 3.0 Unported License.

In recent years, PEDOT:PSS has been studied and proposed as a photothermal agent. Liu and coworkers synthesized poly-(3,4-ethylenedioxythiophene):poly(4-styrenesulfonate) (PEDOT:PSS) nanoparticles that exhibit uniform spherical shape (Cheng et al. 2012). PEGylation was used as a technique to facilitate the incorporation on biological systems. The high optical absorption allowed irradiation at $808 \mathrm{~nm}$ using low laser power density. The authors highlighted the good photostability of the nanomaterial, compared with typical metal nanoparticles. In vivo experiments were carried out by employing Balb/c mice as animal model for PTT, showing excellent therapeutic efficacy. A recent work, showed the synthesis of poly(diethyl-4,4'-\{[2,5-bis(2,3-dihydrothieno[3,4-b][1,4]dioxin-5-yl)-1,4-phenylene] bis(oxy) $\}$ dibutanoate) (P1) and poly(3,4-ethylenedioxythiophene) (PEDOT) nanoparticles where 4-dodecylbenzenesulfonic acid and poly(4-styrenesulfonic acid-co-maleic acid) are used as stabilizers (Cantu et al. 2017). Particles with an average size of $100 \mathrm{~nm}$ were obtained by the two-surfactant emulsion polymerization method. An exhaustive study based on the effect of nanoparticles concentration on PTT activity was carried out. Laser power was fixed on $0.56 \mathrm{~W}\left(2.0 \mathrm{~W} \mathrm{~cm}^{-2}\right)$, and laser irradiation wavelength was $808 \mathrm{~nm}$. Both kinds of nanoparticles studied in this work present important absorption peaks 
in the NIR region of the electromagnetic spectra. In a concentration range between 10-500 $\mu \mathrm{g} \mathrm{mL}^{-1}$, the PTT effect was corroborated in both cases, showing a significant increase in local temperature $\left(\sim 35-40^{\circ} \mathrm{C}\right)$, with a linear relationship between nanomaterial concentration and temperature. Moreover, the photostability of the dispersions allowed the repetition of irradiation cycles (Figure 4). Photothermal conversion efficiency for both kinds of nanoparticles was estimated at $50 \%$. The materials were successfully tried as agents for laser-induced PTT, showing complete cell ablation achieved by the generation of local heat capable to cause the death of MDA-MB-231 cells at low concentrations (c.a. $\left.10 \mu \mathrm{g} \mathrm{mL} \mathrm{L}^{-1}\right)$.
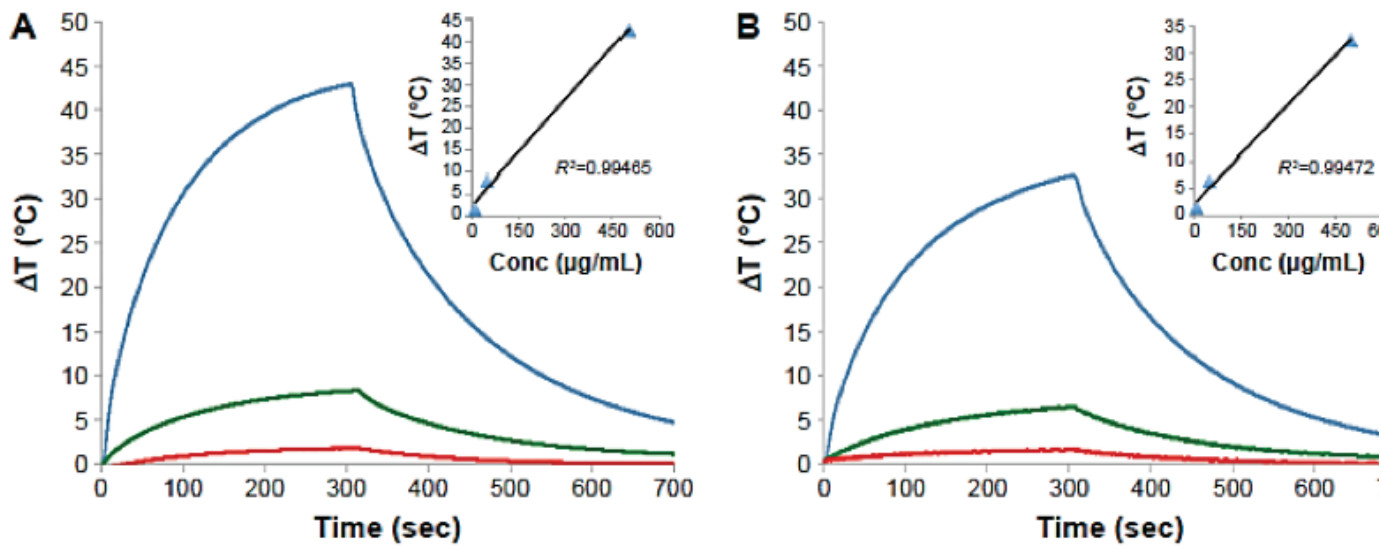

$-500 \mu \mathrm{g} / \mathrm{mL}-50 \mu \mathrm{g} / \mathrm{mL} \quad-10 \mu \mathrm{g} / \mathrm{mL}$
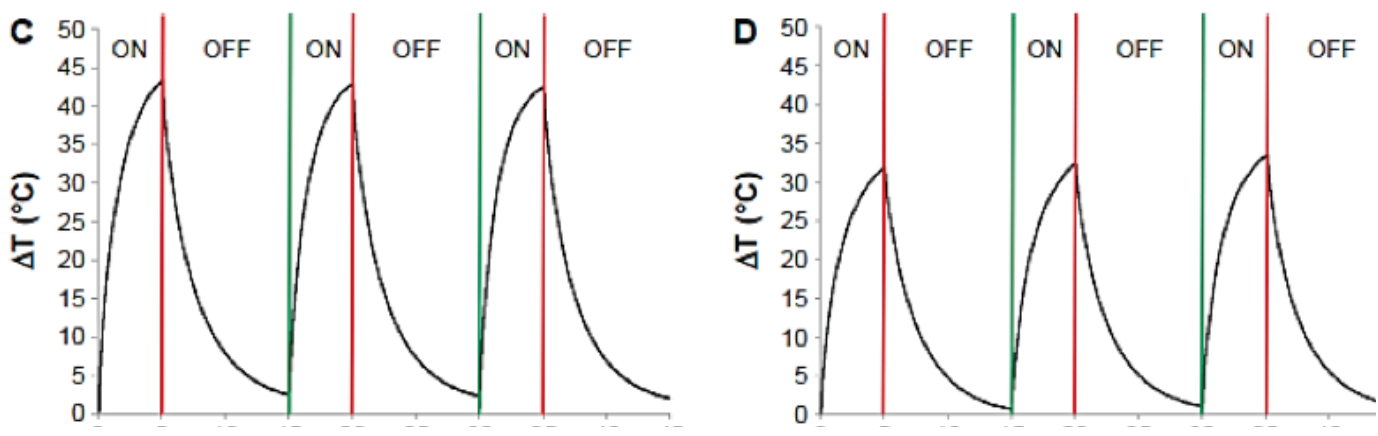

Figure 4. Temperature (T) changes in P1-PMD (A) and PEDOT-PMD (B) NPs at three different concentrations upon irradiation with $808-\mathrm{nm}$ laser at $2 \mathrm{~W} \mathrm{~cm}^{-2}$ for 5 min followed by cooling in the absence of irradiation. Cycled laser-mediated irradiation (ON) and cooling in the absence of irradiation (OFF) for P1-PMD (C) and PEDOT-PMD (D) NP suspensions. Abbreviations: NPs, nanoparticle; P1, poly(diethyl-4,4'- (Zha et al. 2013-b][1,4]dioxin-5-yl)-1,4-phenylene] bis(oxy)) dibutanoate); PEDOT, poly(3,4-ethylenedioxythiophene); DBSA, 4-dodecylbenzenesulfonic acid; PSS-co-MA, poly(4-styrenesulfonic acid-co-maleic acid); P1-PMD, P1:PSS-co-MA:DBSA; PEDOT-PMD, PEDOT:PSS-co-MA:DBSA. Reproduced with permission from reference (Cantu et al. 2017) which is licensed under a Creative Commons Attribution - Non Commercial (unported, v3.0) License.

Composite and nanocomposite materials are nowadays flourishing as potential PPT agents. In this way, different materials have been used for PTT applications combining conducting polymers with other kinds of nanostructures or components, such as biopolymers, metals, and carbon-based materials. For example, gold nanorods combined with poly(o-methoxyaniline) (GNRs/POMA) were reported as a promising material. In this synthetic approach, POMA acts as a coating on the nanogold surface, resulting in a kind of core-shell particle. The conducting polymer helps improve the PTT conversion efficiency of the metal particles. In this way, authors demonstrated the photothermal effect of these hybrid materials by exposition under NIR laser at a power of $3.0 \mathrm{~W} \mathrm{~cm}^{-2}$, performing six cycles of irradiation. Colon cancer CT26 cells were employed for in vitro experiments, showing a significant increase of photothermal efficiency when the polymeric shell is present compared with the GNRs without POMA. Only 10 minutes of laser irradiation were enough for the destruction of tumoral cells in contact with the composite. Besides, typical in vivo assays employing female Bal$\mathrm{b} / \mathrm{c}$ mice were carried out, regarding a successful tumor elimination by using GNRs/POMA core/shell hybrids (Wang et al. 2018). Combining PANI with GO (GO@PANI), PTT nanoagents were obtained as nanosheets hybrids with nanorod array. Under typical conditions of NIR irradiation using a spot size $1 \mathrm{~cm}$ long and $0.5 \mathrm{~cm}$ wide, the results have shown temperature increasing higher than $20^{\circ} \mathrm{C}$ after 700 seconds, with a conversion efficiency close to $50 \%$. This parameter was estimated at $26 \%$ when PANI is not present in the GO system (Fan et al. 2019). Other examples containing PANI for 
applications in antibacterial PTT were summarized by Xu et al., including, among others, NMPA-CS micelles and PANI/ PVP composites (Xu et al. 2019). Recently, poly (3,4-ethylenedioxythiophene):poly(styrene-sulfonate)/agarose nanocomposites were obtained by Ko et al. as antibacterial photothermal materials as hydrogel using a simple synthetic method. Escherichia coli (E. coli) and Staphylococcus aureus (S. aureus) were the bacteria chosen for the antibacterial PTT tests (Ko et al. 2019). The bactericidal action was evaluated at different times in both cases. Under only 1 minute of exposition to NIR light (power $2 \mathrm{~W} \mathrm{~cm}^{2}$ ), the viability of $E$. coli decreased to $44 \%$. In the case of $S$. aureus, viability was reduced to $37 \%$ compared to the original colonies. Additionally, in 2 minutes under the same power laser at $808 \mathrm{~nm}$, both bacteria were totally killed, demonstrating the excellent antibacterial effect of the nanocomposites.

Furthermore, in recent years, a new trend is focusing on nanosystems that exhibit a combined effect of different phototherapies (Pierini et al. 2018). As a result, the effect of PTT and photodynamic therapeutic (PDT) combined were explored by using conducting polymers composites. Poly(pyrrole-3-carboxylic acid) (PPyCOOH) nanoneedles were generated in order to act as an agent on dual-imaging guided PDT/PTT combination therapy. The final material resulted in the complex PPyCOOH nanoneedles modified with poly(allylamine hydrochloride) (PAH) and poly(acrylic acid) (PAA) and loaded with the photosensitizer aluminum phthalocyanine tetrasulfonate (AlPCS $)$ (AlPCS4@PPyCONH-PAH-PAA). Nanoneedle therapeutic performance was highly superior to that reached by nanospheres. The fluorescent properties allowed guiding the nanomaterial used for PDT/PTT chemotherapy on in vitro and in vivo assays (Liu et al. 2018). Using the same conducting polymer (PPy) but combined with bismuth, a multifunctional nanocomposite was synthesized as a core-shell hybrid by Yang et al. for anticancer treatment (Yang et al. 2018). In vitro and in vivo assays demonstrated a highly effective photothermal ablation on cancer cells that can be combined with computed tomography and photoacoustic dual modal imaging in order to improve the diagnosis. Cao et al. developed a system that presents photoacoustic imaging (PAI)-guided photothermal/chemotherapy (Cao et al. 2017). Diketopyrrolopyrrole-based polymers (DPP) were designed by combining donor-acceptor moieties. Encapsulation of doxorubicin (DOX) and DPP in tocopherol polyethylene-glycol-succinate-cholesterol (TPGS-CHO) copolymers were made in order to generate the theranostic platform for cancer treatment. NIR-II photothermal nanoagents based on a conjugated polymer (TBDOPV-DT) with 2,2-bithiophene as the donor and thiophene-fused benzodifurandione-based oligo( p-phenylenevinylene) as the acceptor were produced by nanoprecipitation and reported by Sun et al (Sun et al. 2018). Their study showed the application of composite nanoparticles for PAI and PTT. Under $1064 \mathrm{~nm}$ (NIR-II) laser irradiation, a 50\% efficiency in PTT conversion was successfully achieved. The material is promissory for tumoral treatments considering the effect of PTT and the ability of PAI for imaging guiding.

\subsection{Metallic nanoparticles}

In the past decades, nanoparticles have been exploited in many biomedical applications (Thota et al. 2018), some of which have paid special attention to noble metal nanoparticles applied in PTT, especially gold (Au) nanoparticles (Boisselier et al. 2009). This is a consequence of their unique optical and photothermal properties, including strong optical absorption and a relatively high photothermal conversion efficiency, showing great photostability. However, other metallic nanoparticles have also been investigated as photothermal agents: palladium nanoparticles, silver nanotriangles, and copper nanoparticles.

Undoubtedly, gold nanoparticles are one the most studied and applied nanomaterials in PTT, mainly because of their simple synthesis and surface modification process, the varied forms that can be manufactured, and their biocompatibility. Simple spherical Au nanoparticles have been widely studied but offer very limited tunability of their "localized surface plasmon resonance" (LSPR) frequency. While a red shift of LSPR is possible, it has been shown that changing the size of Au nanospheres from $9 \mathrm{~nm}$ to $99 \mathrm{~nm}$ only changes LSPR from 520 to $580 \mathrm{~nm}$ (Link et al. 1999). Therefore, none are suitable for NIR applications. However, nanoparticle shape has also a strong influence on the LSPR effect, and LSPR could be shifted to the NIR region by producing metallic nanoparticles with non-spherical shapes such as nanorods, nanocages, nanoshells, nanostars, and even nanobipyramids (Lombardi et al. 2012, Wang et al. 2013). The development of these different gold nanomaterials allowed achieving photothermal performance by simply tuning the LSPR by changing nanomaterial shap.

Among the mentioned non-spherical gold nanoparticles, nanorods (GNRs) have attracted a great deal of attention due to the large number of synthesis methods available, the high achievable monodispersity, and the rational control over the aspect ratio (length vs. diameter), which is primarily responsible for the change in their optical properties. GNRs have two characteristic optical absorptions, transversal and longitudinal, linked to rod the diameter and lengt, respectively. In GNRs spectra (Figure 5a), it can be seen how the LSPR band is split into two bands - a strong band in the NIR region, corresponding to electron oscillations along the long axis (longitudinal band), and a weak band in the visible region at a wavelength similar to that of gold nanospheres (transversal bands). Link et al. studied the relationship between the absorption maximum in the longitudinal band and particle aspect ratio (Link et al. 2005). According to their research, the 
transversal band is insensitive to size changes, whereas the longitudinal band is red-shifted largely from the visible to NIR region with increasing aspect ratios (length/width), causing the color changes from blue to red (Figure 5b). Currently, aspect ratio can be precisely controlled by changing experimental parameters such as the catalyst of silver ions in the seed-mediated growth method (Nikoobakht et al. 2003).

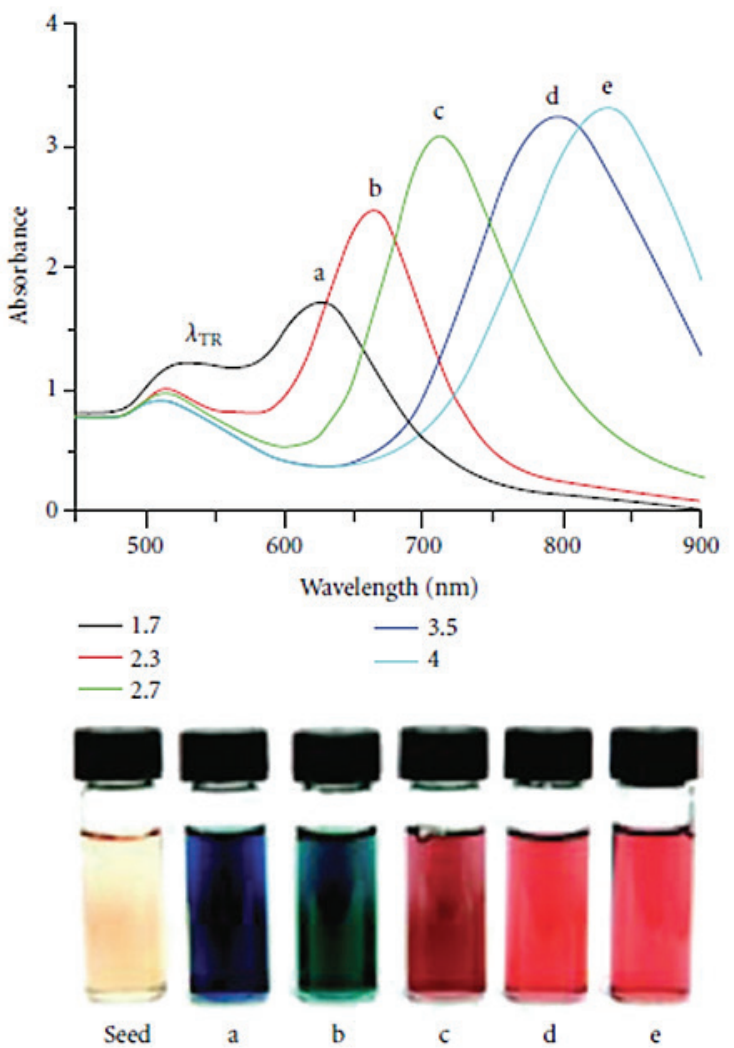

(a)

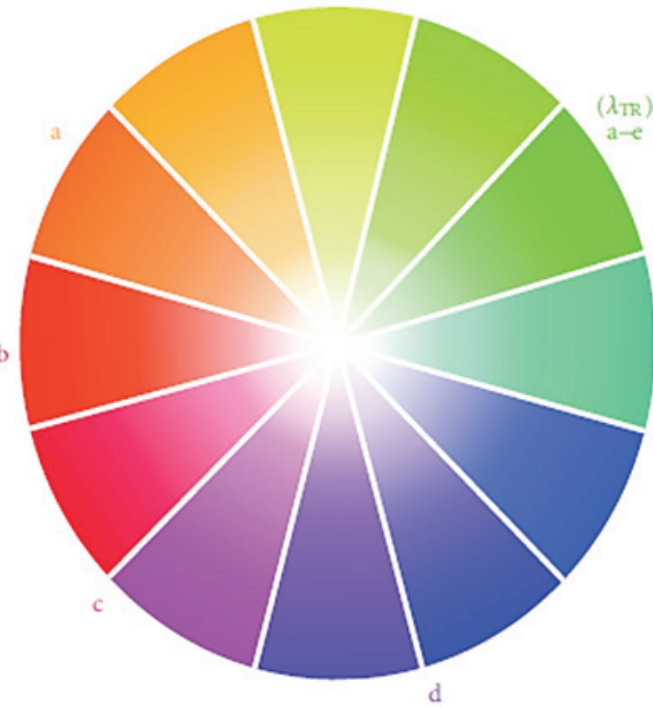

(b)

Figure 5. Tunable optical properties of gold nanorods (AuNRs) with absorptions at visible and near-infrared wavelengths region. (a) Optical absorption spectra of AuNRs with different aspect ratios and composition (a-e). (b) Color wheel representing AuNRs labeled a-e, TR $=$ transverse resonance. Reproduced with permission from reference (Tong et al. 2009) with permission from John Wiley and Sons and Copyright Clearance Center.

Nanoshell structures are another example of gold nanoparticles that absorb in the biological window and can be used as PTT agents (Jain et al. 2007). In this case, a broad plasmonic extinction band is observed that can be tuned by varying core and shell thickness, as well as total nanoparticle size (Jain et al. 2006). Loo et al. demonstrated that the plasmonic band shifts to the red when shell thickness decreases (Loo et al. 2004).

El-Sayed and coworkers firstly demonstrated the in vitro application of gold nanorods in PTT (Huang et al. 2006). They studied the binding of the gold nanorods conjugated to anti-epidermal growth factor receptor antibodies, specifically to the malignant epithelial cell lines. After applying the PTT treatment using a Ti:Sapphire laser at $800 \mathrm{~nm}$ for $4 \mathrm{minutes}$, they found that cancer cells required half the laser energy $\left(10 \mathrm{~W} \mathrm{~cm}^{-2}\right)$ to be photothermally damaged, compared to normal cells $\left(20 \mathrm{~W} \mathrm{~cm}^{-2}\right)$. This was attributed to the presence of nanoparticles on cell surface. Comparing these results with the ones obtained by Hirsch et al. with gold nanoshells (Hirsch et al. 2003), where they found that breast carcinoma cells incubated with PEGylated gold nanoshells underwent irreversible photothermal damage after exposure to a NIR laser (820 $\mathrm{nm}, 35 \mathrm{~W} \mathrm{~cm}^{-2}$ ) for 7 minutes, using gold nanorods enables effective treatment at three times lower laser intensity. This is because nanorods exhibit higher absorption efficiency than nanoshells with LSPR at the same wavelength. Thus, it is possible to achieve effective treatment with lower laser intensity using GNRs as photothermal agents.

In addition to the application of PTT for cancer therapies, gold nanoparticles have attracted special attention in researchers who are investigating alternative treatments for bacterial infections, especially those caused by drug-resistant bacteria (Xu et al. 2019). Zharov et al. proposed that hyperthermia caused by gold-based metallic nanoparticles could be a useful mechanism to kill pathogenic bacteria combining the use of laser and functional gold nanoparticles (Zharov et al. 
2006). When the nanoparticles reach their target cells and this is followed by irradiation with NIR light, the overheating effects could destroy the target cells rapidly. In addition, some research groups investigated the combination of different methods to achieve more efficient and more specific antibacterial activities in gold nanostructures, like the incorporation of antibiotics for instance. Huang and coworkers studied the use of gold nanoparticles with polygonal shapes functionalized with the antibiotic vancomycin (Au@van nanoparticles) as photothermal agents, when combined with NIR light irradiation ( $808 \mathrm{~nm}, 0.2 \mathrm{~W} \mathrm{~cm}^{-2}$ ), Au@van nanoparticles could effectively inhibit pathogenic bacteria cell growth within 5 minutes, including Gram-positive, Gram-negative, and antibiotic-resistant bacteria (Huang et al. 2007). Moreover, Chen et al. tested the synergistic effect of photothermal treatment and antibiotic killing of S. aureus (Meeker et al. 2016). They fabricated gold nanoconstructs coated with polydopamine (PDA) for loading antibiotic daptomycin (Dap) (Figure 6). These Dap-loaded nanostructures were targeted to the bacteria by conjugating antibodies against staphylococcal protein A. The targeted nanodrug was activated by NIR light to convert the photo-energy to thermal energy; the resulting temperature change caused the simultaneous generation of localized photothermal effects and PDA expansion, leading to controlled antibiotic release. It was shown that it is possible to enhance the antibacterial activity of gold nanoparticles by combining them with many other materials. Therefore, this method has the potential for use as a therapeutic method for biofilm-associated infections caused by drug-resistant bacteria.
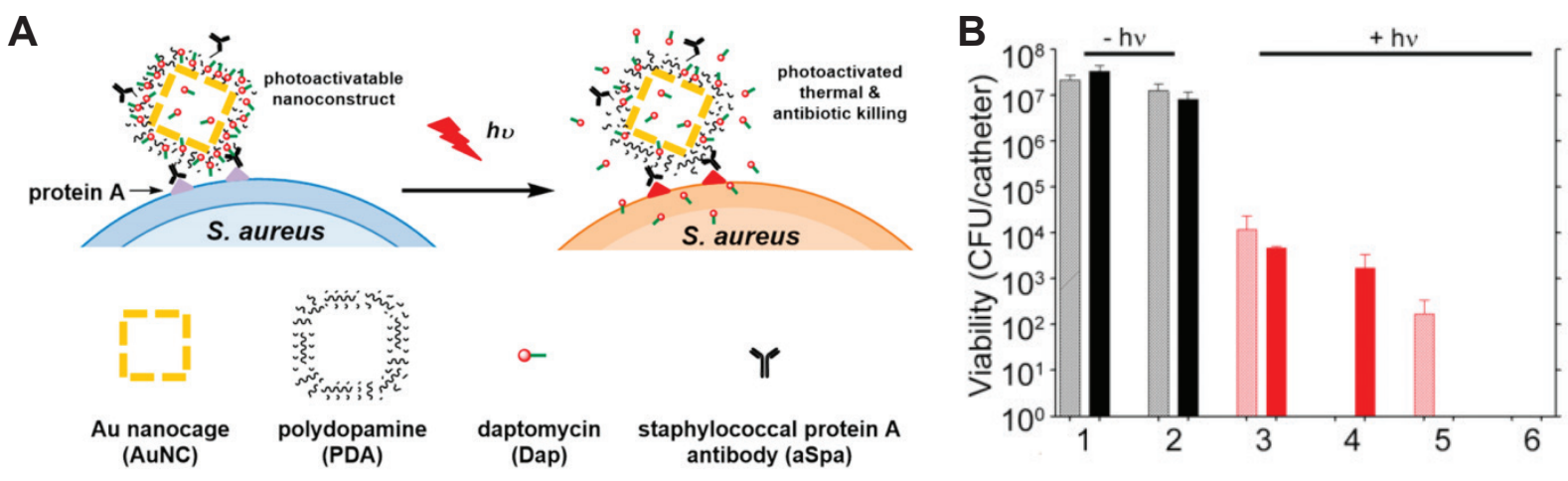

Figure 6. A) Schematic illustration of the working mechanism in the targeted photoactivatable nanoconstruct for the synergistic photothermal and antibiotic treatment of $S$. aureus. B) Bacterial cell killing using a biofilm model. Experimental groups are (1) no treatment, (2) $5 \mu \mathrm{g} \mathrm{mL}^{-1}$ daptomycin (Dap), and irradiation plus (3) polydopamine-coated gold nanocages (AuNC@PDA), (4) AuNC@PDA conjugated to staphylococcal protein A; Spa (aSpa), (5) AuNC@PDA loaded with Dap (AuNC@Dap/PDA), and (6)AuNC@DapHi/PDA conjugated to aSpa (AuNC@Dap/PDA-aSpa).Killing was assessed at $0 \mathrm{~h}$ (striped bars) and $24 \mathrm{~h}$ (solid bars) after treatment. Black bars indicate non-irradiated groups, and red bars indicate irradiated groups. This figure has been adapted from reference (Meeker et al. 2016) which was published under an ACS AuthorChoice License.

Another interesting example of the application of PTT in bacterial infection treatment is a hybrid system of Au NPs in conjunction with polydopamine-assisted hydroxyapatite prepared by Xu et al. (Xu et al. 2018). This study presented in situ promotion of wound healing by a low-temperature PTT assisted nanocatalytic antibacterial system utilizing a polydopamine (PDA) coating on hydroxyapatite (HAp) incorporated with gold nanoparticles (Au-HAp). The PDA@AuHAp NPs produce hydroxyl radicals $(\bullet \mathrm{OH})$ from $\mathrm{H}_{2} \mathrm{O}_{2}$ at low concentrations, thus enhancing photothermal antibacterial performance at $45^{\circ} \mathrm{C}$ and avoiding unnecessary damage on normal tissues. In addition, this approach stimulated tissue repairing-related gene expression to facilitate the formation of granulation tissues and collagen synthesis, accelerating wound healing.

Although noble metal nanomaterials have offered an outstanding photothermal effect for different medical issues, such as cancer treatment and bacterial infections, some problems may need to be solved. For instance, gold-based nanomaterials are non-biodegradable, which leads to further detection of nanoparticles after treatment. Therefore, it is necessary to investigate new kinds of nanomaterials in order to develop photothermal agents with exceptional optical properties, good biocompatibility, and that are biodegradable.

\subsection{Carbon-based nanoparticles}

The noble metal nanomaterials mentioned before may present some problems that have significantly limited their application in clinical trials, such as their non-biodegradability and possible long-term cytotoxicity. To achieve excellent photothermal nanoagents with improved biocompatibility and lowered cytotoxicity, carbon-based nanoparticles have been 
widely studied in recent years in order to reduce lateral effects, mainly due to the intrinsic biocompatibility of carbon. Among the extensive range of carbon nanomaterials, we will focus on graphite-related structures such as single and multiwall carbon nanotubes (SWCNT and MWCNT, respectively), and graphene derivatives, including graphene oxide (GO) and reduced graphene oxide (rGO). Some applications of nanodiamonds in PTT will also be mentioned.

In the last decades, carbon nanotubes (CNTs) have been the most developed carbon-based nanostructures. These nanostructures, discovered by Iijima (Iijima 1991), are allotropic forms of carbon obtained by rolling up graphene sheets into a tube shape, forming a cylinder (Figure 7a) with characteristic diameters ranging from 0.4 to $50 \mathrm{~nm}$. Length, on the other hand, varies greatly with synthesis conditions and can reach values as high as several centimeters (Wang et al. 2009). As a result, CNTs have extremely high aspect ratios. Depending on the number of sheets rolled into concentric cylinders, they can be divided into two broad categories: single-wall carbon nanotubes (SWCNT) and multi-wall carbon nanotubes (MWCNT) (Figure 7b). MWCNTs usually have larger diameters (> $100 \mathrm{~nm}$ ) than SWCNT, which can be as small as $1 \mathrm{~nm}$.

(a)

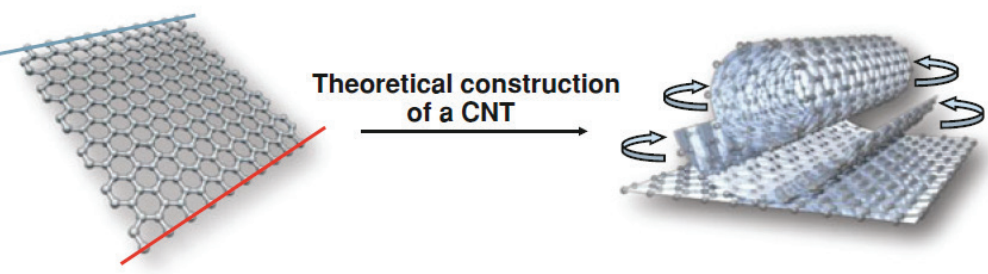

(b)

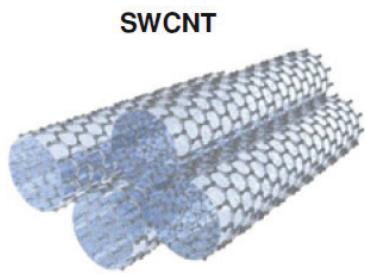

(c)

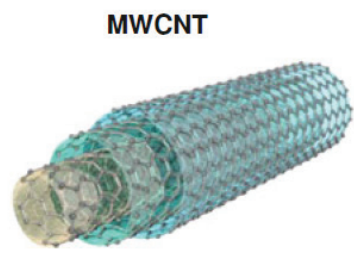

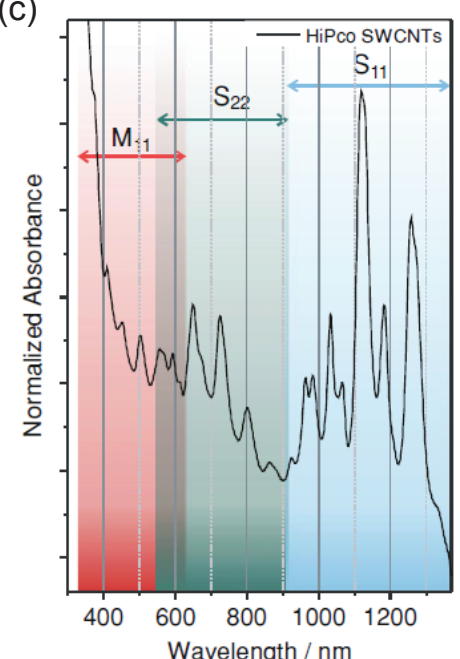

Figure 7. a) Schematic representation of the theoretical construction of a carbon nanotube by rolling up a graphene sheet. b) Representation of a single-walled carbon nanotube (SWCNT-left) and a multi-walled carbon nanotube (MWCNTright). c) Typical UV/Vis/ HiPco SWCNTs nIR absorption spectrum of HiPco SWCNT dispersed in an aqueous solution of SDBS (1 wt $\%$ ) after ultracentrifugation. Metallic $\mathrm{M}_{11}$ transitions are indicated by the red shaded background, and semiconducting $\mathrm{S}_{22}$ and $\mathrm{S}_{11}$ transitions by the green and blue colored shaded background, respectively. Figures have been adapted from reference (Backes et al. 2010) with permission from John Wiley and Sons and Copyright Clearance Center.

CNTs exhibit strong absorbance in the NIR, which makes them a promising tool in PTT (Figure 7c). Typically, this kind of nanomaterials are functionalized with PEG coatings, since this helps improve biocompatibility, prolongs blood circulation time, and avoids aggregation. As a typical example, Kam et al. have demonstrated that, when irradiated by NIR light, CNTs that were internalized in cells can trigger endosomal rupture and cell death without harming normal cells (Kam et al. 2005). This discovery led to the development of SWCNT functionalization whit specific ligands for recognizing and targeting tumoral cells. They exploited folate receptors (FR) that are common tumor markers by making highly water-soluble specific SWCNTs non-covalently functionalized by phospholipid-PEG-folic acid (PL-PEG-FA). After incubating both, normal cells and FR-positive HeLa cells (FR ${ }^{+}$cells), and irradiating them with an $808 \mathrm{~nm}$ laser (1.4 $\mathrm{W} \mathrm{cm}{ }^{-2}$ ) for 2 min, general cell death was confirmed for the $\mathrm{FR}^{+}$cells by cell morphology changes, whereas normal cells remained intact. This selective destruction of $\mathrm{FR}^{+}$cells implied that PL-PEG-FA-SWCNT were successfully internalized inside cancerous cells. In addition to this, Zhang and coworkers reported a self-amplified drug delivery system for tumor PTT using MWCNT, with favorable photothermal effect as the vector, polyethylene glycol (PEG) as the shelter, CREKA peptide as the targeting moiety and NIR illumination as the external power (Zhang et al. 2016). This self-amplified targeting system (MWCNT-PEG) showed strong tumor targeting ability and powerful photothermal therapeutic effectiveness. Moreover, Sobhani et al. were able to improve the dispersability of MWCNTs in water by oxidizing them (O-CNT) and using polyethylene glycol (PEG) for wrapping the surface of nanotubes (Sobhani et al. 2017). They found through different cytotoxicity studies that O-CNT was less cytotoxic than pristine MWCNT, and O-CNT-PEG had the lowest toxicity against HeLa and HepG2 cell lines. The effect of O-CNT-PEG in the reduction of melanoma tumor size after PTT was evaluated by exposing cancerous mice to a continuous-wave NIR laser diode $\left(\lambda=808 \mathrm{~nm}, \mathrm{P}=2 \mathrm{~W}\right.$ and I $\left.=8 \mathrm{~W} \mathrm{~cm}^{-2}\right)$ for 10 
minutes. It was found that tumor average size in the mice receiving O-CNT-PEG decreased sharply in comparison with those that only received laser therapy. Furthermore, using carbon nanotubes as photothermal agents for bacteria killing, Yang et al. designed nonchemotherapeutic and robust dual-responsive nanoagents to control the trapping, ablation, and releasing activities of pathogenic bacteria via an NIR and magnetic stimulus-responsive regulatory nanosystem (Yang et al. 2018). In this system, the temperature-sensitive polymer brush PNIPAm is chemically grown onto the surface of carbon nanotube- $\mathrm{Fe}_{3} \mathrm{O}_{4}$, whereby the synthesized nanoagents $\left(\mathrm{Fe}_{3} \mathrm{O}_{4}\right.$-CNT-PNIPAM) could transition from hydrophilic dispersion to hydrophobic aggregation upon NIR irradiation, which thus controls bacterial trapping, killing, and detaching (Figure 8).

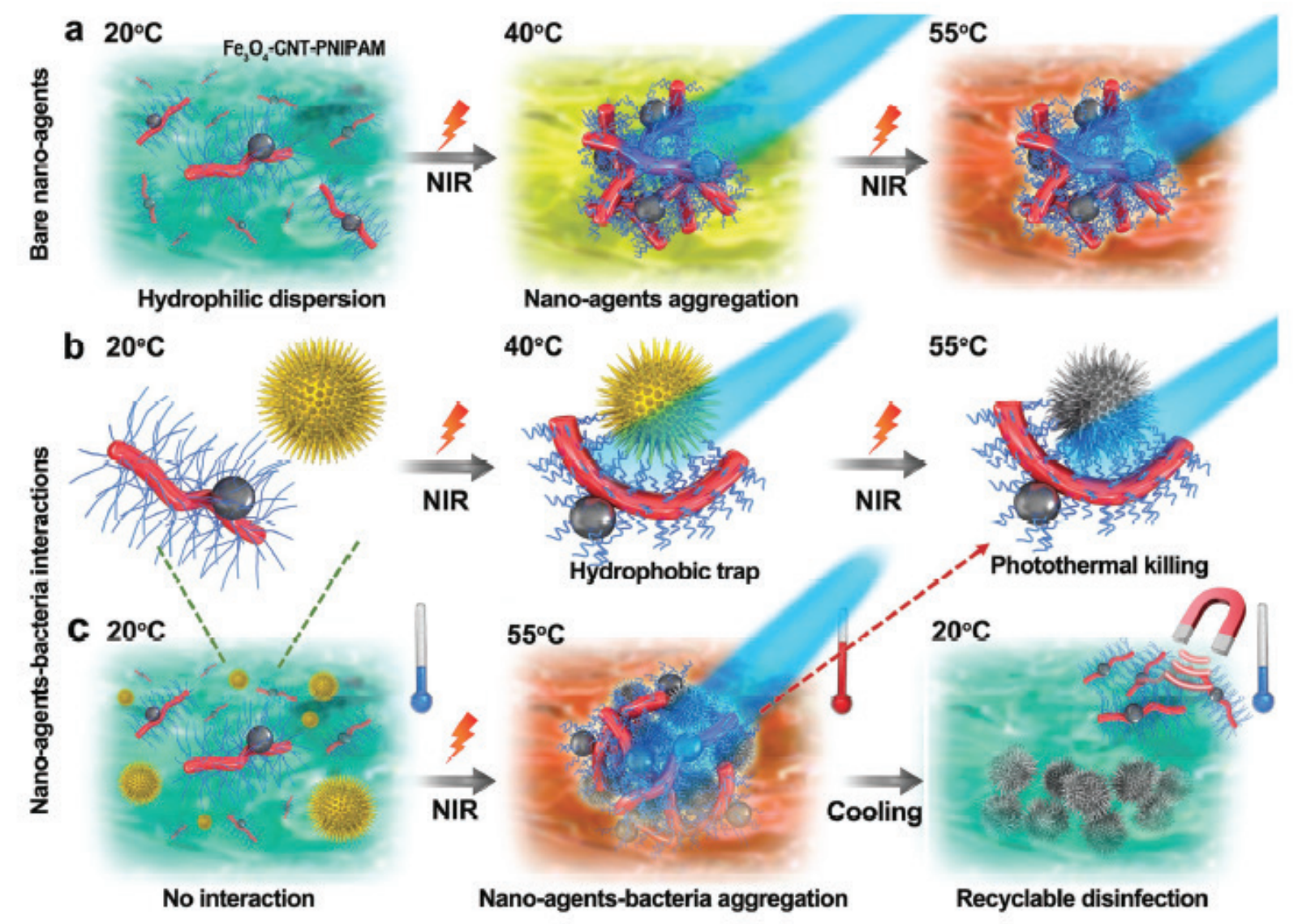

Figure 8. a) The synthesized $\mathrm{Fe}_{3} \mathrm{O}_{4}$-CNT-PNIPAM nanoagents are hydrophilic and well dispersed at $20^{\circ} \mathrm{C}$; upon NIR irradiation, temperature increases; once temperature is above $40^{\circ} \mathrm{C}$, the nanoagents turn completely hydrophobic and form robust aggregations. b) The detailed interactions between nanoagents with bacteria at different temperatures, the bacteria is trapped by the nanoagents at $40^{\circ} \mathrm{C}$, and is killed by the nanoagents at $55^{\circ} \mathrm{C}$. c) The formation of nanoagent-bacteria aggregations and NIR killing of bacteria at $55^{\circ} \mathrm{C}$, the inactivated bacteria can be released from the trap after cooling to low temperature, and the nanoagents can be magnetically collected and reused for antibacterial tests. The figure has been adapted from reference \{Yang, 2018 \#66 $\}$ with permission from John Wiley and Sons and Copyright Clearance Center.

Due to their unique intrinsic physical and chemical properties, which include large surface areas, the ability to penetrate biological membranes, a relatively low toxicity, and special electrical, thermal and spectroscopic properties, CNTs constitute a promising nanomaterial for medical applications. Therefore, CNTs combined with different molecules, including the incorporation of antibiotics, antibodies, metals, and magnetic nanoparticles or tumor markers as well as the functionalization with many polymeric materials, enhance their activity as effective photothermal nanoagents in cancer therapy (Thakare et al. 2010) and bacterial infectious diseases treatment (Xu et al. 2019).

Like CNTs, graphene-based nanomaterials also have strong optical absorption in the NIR region. Compared to metallic nanoparticles and CNTs, graphene materials (especially GO) possess improved properties such as greater optical absorption in the NIR region and higher photothermal conversion, high specific area, and lower cost. Loh and coworkers (Loh et al. 2010) have also found that visible and NIR fluorescence of GO can be tuned depending on the intended application, although it was impossible to fully understand the mechanism. Graphene derivatives such as pristine (non-oxi- 
dized) graphene sheets, GO and rGO have been widely studied for various biomedical applications, such as drug delivery system, cell imaging, and PTT. For instance, Yang et al. were the first group in investigating the application of graphene in PTT (Yang et al. 2010). They found that nanographene sheets (NGS) functionalized with PEG (NGS-PEG) exhibited strong NIR absorbance and in vivo efficient tumor destruction under NIR laser irradiation. Additionally, the studied nanomaterial presented relatively low retention in reticuloendothelial systems. Furthermore, Dai and colleagues (Robinson et al. 2011) developed nanosized, reduced graphene oxide (nano-rGO) sheets with high NIR light absorbance and biocompatibility functionalized with PEG and carrying a targeting peptide covalently bonded. This nanomaterial demonstrated to be an efficient photothermal agent, since it caused a highly effective in vitro photoablation of cells.

In addition, combining photothermal and chemotherapy to generate a synergistic treatment is a more effective way to destroy cancer cells than monotherapy. Chen et al. studied the combination of rGO with gold nanoparticles and produced a hybrid reduced rGO-loaded ultrasmall gold nanorod vesicle (rGO-AuNRVe) with remarkably amplified PA performance and photothermal effects (Song et al. 2015). The hybrid is an excellent drug carrier due to its high loading capacity of doxorubicin (DOX), as both the cavity of the vesicle and the large surface area of the encapsulated rGO can be used for loading DOX. The loaded drug is released sequentially - NIR photothermal heating induces DOX release from the vesicular cavity (Figure 9i), and an intracellular acidic environment induces DOX release from the rGO surface (Figure 9ii).

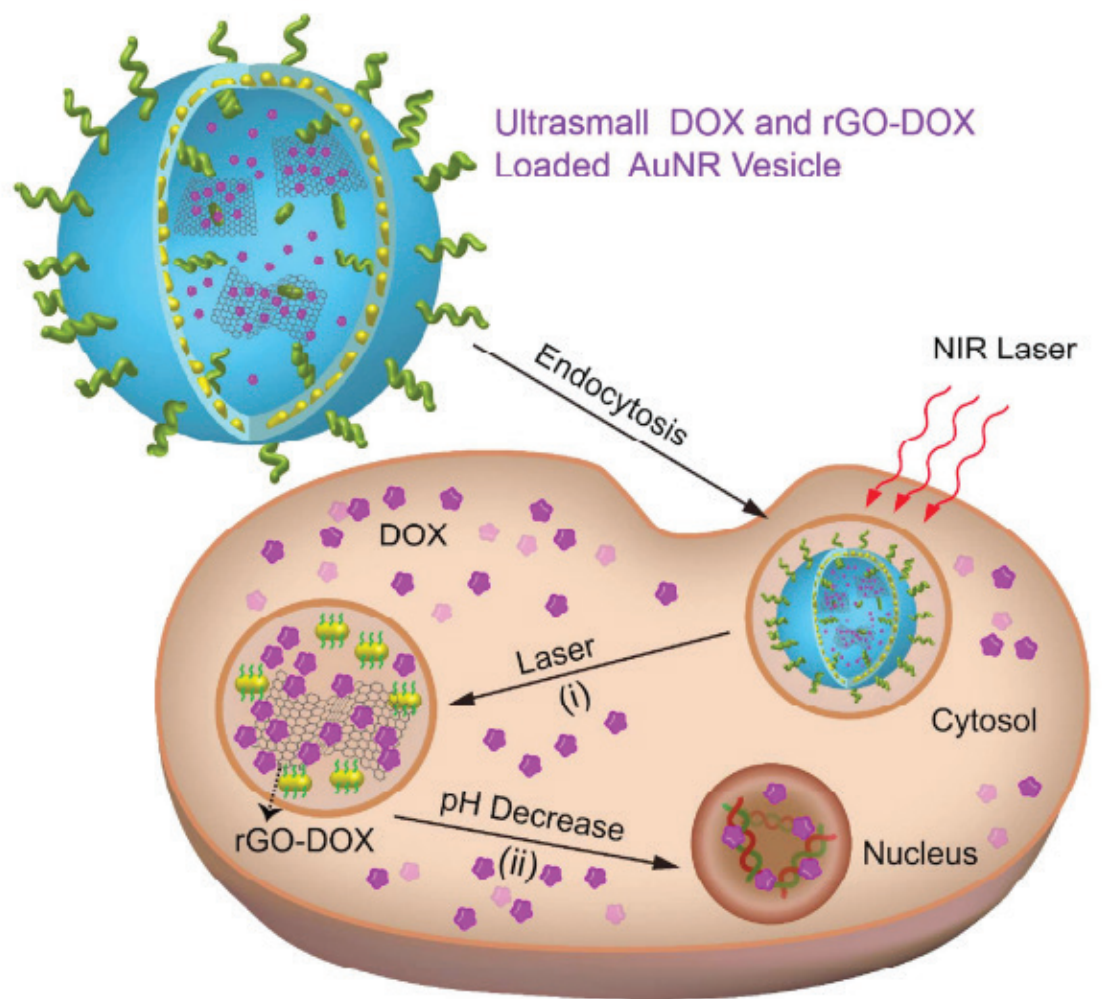

In Vitro Sequential Drug Release

Figure 9. Schematic illustration of sequential DOX release triggered by (i) remote NIR laser irradiated photothermal effect and (ii) acidic environment of the cancerous cell. Adapted from reference (Song et al. 2015) with permission from the American Chemical Society.

Furthermore, graphene nanomaterials are also being studied in combination with different nanomaterials in PTT applied to the treatment of infectious diseases caused by drug-resistant bacteria (Xu et al. 2019). Recently, Pereyra et al. (Pereyra et al. 2018) demonstrated that GO dispersion at low concentrations (c.a. $2 \mu \mathrm{g} \mathrm{mL} \mathrm{mL}^{-1}$ ) under NIR irradiation for 15 min decreased bacterial viability by $\sim 98 \%$. P. aeruginosa treated with GO and irradiated exhibited DNA fragmentation due to the physical damage of cell membranes. This characteristic represents an advantage in comparison with traditional antibacterial nanomaterials (Ag NPs, for instance), which induce cell killing due to the toxicity of the nanoparticles per se. Feng and colleagues investigated 2D rGO supported Au nanostar nanocomposite (rGO/AuNS) for synergistically killing multidrug-resistant bacteria (Feng et al. 2019). Owing to their prickly and sharp-edge nanostructure, rGO/AuNS displayed superior antibacterial activity probably due to the damaging of the cell walls or membranes. It was found that the bactericidal efficiency was significantly enhanced when irradiated with $808 \mathrm{~nm}$ NIR laser, mainly due to the localized 
hyperthermal effect of rGO/AuNS. (Figure 10) Thus, the production of AuNS and rGO nanocomposites is an alternative potential photothermal agent for the synergistic killing of bacteria.

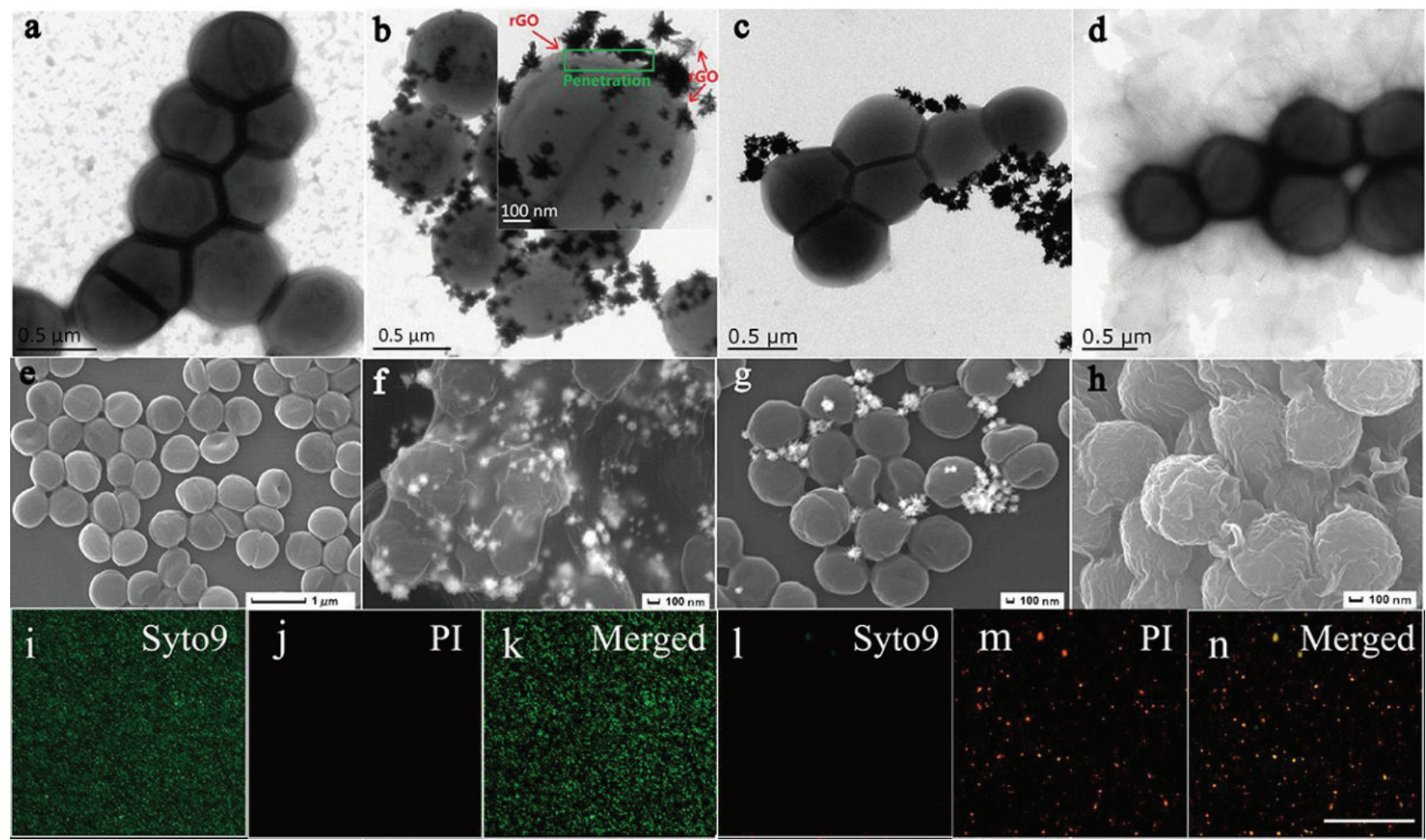

Figure 10. TEM images of MRSA (a), MRSA incubated with rGO/AuNS0.02 (b) and AuNS (c) after $808 \mathrm{~nm}$ NIR irradiation for $6 \mathrm{~min}$, and MRSA incubated with rGO (d); SEM images of MRSA (e), MRSA incubated with rGO/AuNS0.02 (f) and AuNS (g) after $808 \mathrm{~nm}$ NIR irradiation for $6 \mathrm{~min}$, and MRSA incubated with rGO (h). Laser power density was 3 $\mathrm{W} \mathrm{cm}{ }^{-2}$. Fluorescence microscopy images of MRSA (i), (j) and (k) and MRSA exposed to NIR laser irradiation for 6 min after incubating with $0.26 \mathrm{mM} \mathrm{rGO/AuNS} 0.02$ (I), (m) and (n), which were treated with LIVE/DEAD BacLight Bacterial Viability Kit. The scale bar in the figure is $100 \mu \mathrm{m}$. The figure has been adapted with permission from Reduced Graphene Oxide Functionalized with Gold Nanostar Nanocomposites for Synergistically Killing Bacteria through Intrinsic Antimicrobial Activity and Photothermal Ablation. ACS Applied Bio Materials. 2(2):747-756. Copyright 2019 American Chemical Society.

The works mentioned here provided an innovative treatment for combating bacterial nosocomial infections without the use of antibiotics, opening a new area of clinical application via simple PTT.

Finally, nanodiamonds (NDs) with a bulk diamond-like structure have emerged as promising carbon nanomaterials in biomedical applications mainly due to their high surface area, optical properties, high biocompatibility, nontoxicity, colloidal stability, excellent mechanical strength, and high surface functionality. Generally, NDs have been used as luminescent biomarkers capable of intracellular high-resolution tracking, and recently have been proposed as excellent nanothermometers that can be used for thermal imaging of single cells (Kucsko et al. 2013). However, these nanomaterials have not shown high light-to-heat conversion efficiencies; thus, they are not suitable for photothermal applications. Ahn et al. deposited polyaniline on diamond nanoparticles (PANI-ND) by polymerizing aniline at the surface of NDs for efficient PTT (Ahn et al. 2019). They found that the UV absorbance intensity of PANI-ND increased at the lower pH in the NIR region, as expected for PANI layers, resulting in an enhanced photothermal effect at the tumor site. Notably, the viability of HeLa cells treated with PANI-ND decreased by less than $20 \%$, suggesting the high efficiency of PTT using this functionalized nanodiamons. Therefore, it is likely that NDs act as nanotemplates to form core-shell (ND/PANI) nanoparticles.

\subsection{Other nanomaterials and composites using dyes for PTT}

As mentioned in previous sections, PTT is now combined with other therapies that also involve a light process in order to enhance efficiency. In this sense, the use of organic dyes facilitates and gives new opportunities to achieve successful 
treatment. In this section, some examples of recently published works involving dyes incorporation or conjugation with diverse nanomaterials/composites for combined therapies are described.

Wang et al. summarized the research focused on the photothermal generation conjugating organic colorant dyes with different nanostructures (e.g. nanoparticles, polymeric micelles, block copolymers, among others) (Wang et al. 2016). The most relevant dyes involve cyanine derivates and the well know IR-780, IR-820, and IR-825 dyes. In all cases, irradiation was applied at $808 \mathrm{~nm}$. Different cancer cell types and animal models for in vitro and in vivo assays, respectively, were employed. Additionally, using indocyanine, multifunctional nanoparticles based on Pt(II)-meso-tetra-(pentafluorophenyl)porphyrine (PtTFPP) and poly(9,9-di-noctylfluorenyl-2,7-diyl) (PFO) loaded with indocyanine green (ICG), a novel material was fabricated (Wang et al. 2017). The nanomaterial involves an organic polymer (PFO), a photosensitizer porphyrin (PtTFPP), and a NIR absorbing dye (ICG). This particular design enables a strong photothermal absorption under laser light added to the high singlet oxygen generation, being a very interesting agent for applications on tumoral therapies combining PTT with PDT. The authors remark that a synergistic effect is present, improving the therapeutic efficacy of both individual therapies (PTT or PDT). In the same way, heptamethine cyanines (IR-825, IR-780, IR-808, and IR-2) were proposed as dyes for conjugation with polymeric micelles for PTT activity. The use of these organic compounds was excellently summarized by Jung et al. (Jung et al. 2018) (Figure 11). In addition, they have shown many other possibilities - for example, the application of diverse croconaine, porphyrins, and diketopyrrolopyrrole-based agents for photothermal effect - with successful results towards new platforms that complement PTT with PAI, NIR fluorescence imaging, and MRI, among others.

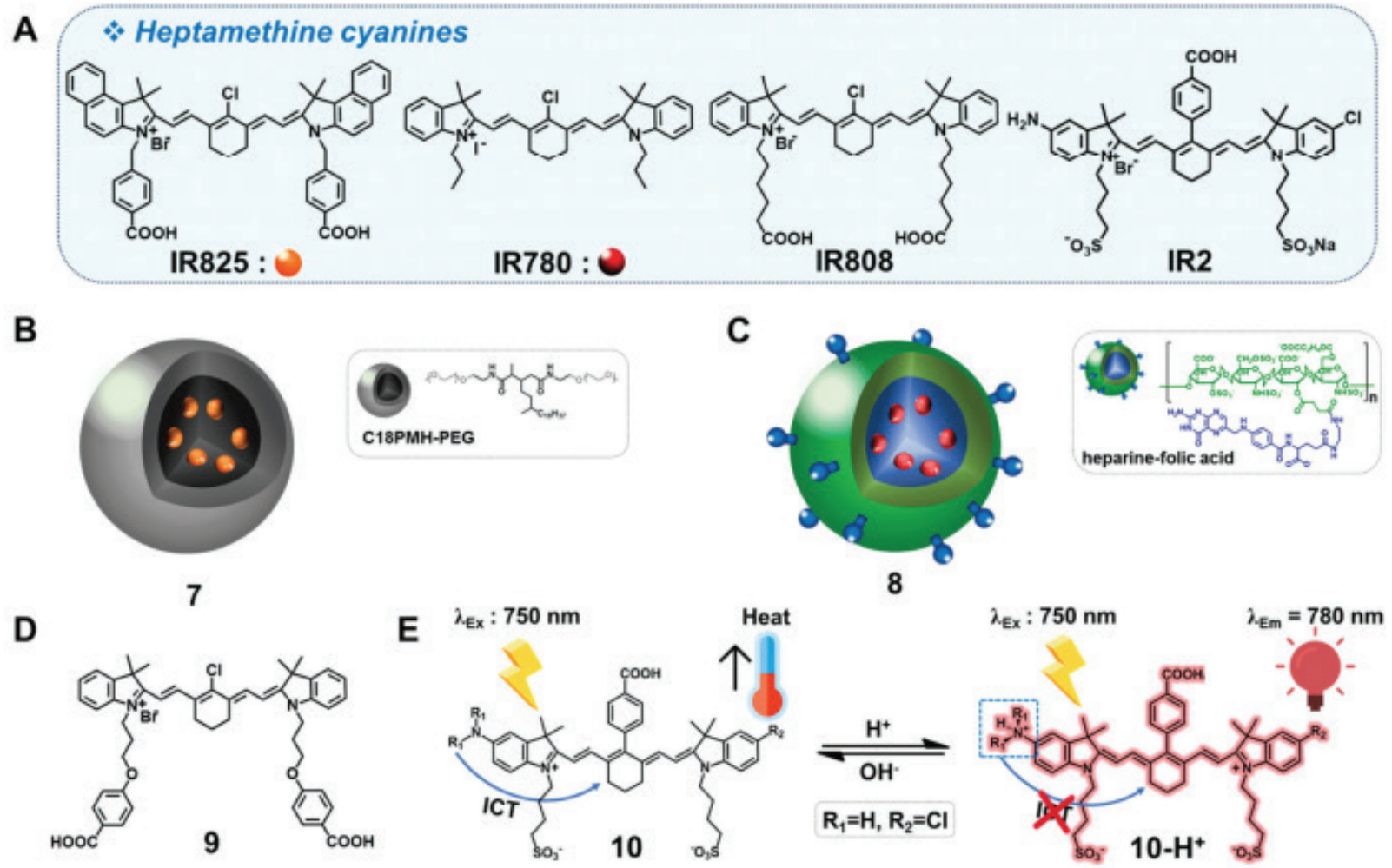

Figure 11. (A) Molecular structures of the heptamethine cyanines: IR825, IR780, IR808, and IR2 and associated PTT systems: (B) IR825/PEGylated micelle 7, (C) IR780/heparine-folic acid system 8, (D) mitochondria-targeted heptamethine 9, and (E) pH-switchable theranostic heptamethine dyes 10 and 10-H+. This figure has been reproduced from reference $\{$ Jung, $2018 \# 100\}$ with permission from the Royal Society of Chemistry.

Another material reported for NIR imaging and PTT were heparine-folic acid nanoparticles loaded with IR-780 (HFIR-780 NPs). The authors affirm that IR-780 exhibits some advantages compared to ICG, such as stability and fluorescence intensity. Dye conjugation was made by ultrasonication process, obtaining monodisperse spherical particles. MCF7 cells were employed for in vitro assays, and it was concluded that nanomaterial does not present cytotoxic effects and is biogradable. The results of these experiments reveal a temperature increase in MCF7 tumor tissues of $20 \mathrm{C}$, making HFIR-780 NPs a promising photothermal nanoagent and a potential candidate for theranostics (Yue et al. 2013). On the other 
side, Calderón and coworkers reported the design of a system that involves a smart thermoresponsive nanogel scaffold that allows obtaining a combination of PDT/PTT. The synthetic approach used the covalent conjugation by amide formation between acrylated polyglycerol amine (dPG-Ac) and the dye (IR-806) for smart nanogel production (tNG-IR-806), exhibiting controlled shape, size, and monodispersity (c.a. $90 \mathrm{~nm}$, PDI: 0.16). By heating on the tNG-IR806 at different concentrations using a NIR irradiation source (wavelength: $785 \mathrm{~nm}$, power density: $0.59 \mathrm{~W} \mathrm{~cm}^{-2}$ ), thermal collapse was evidenced by turbidimetric experiments in several cycles. (Figure 12). Upon NIR laser irradiation, a hyperthermia process was observed with the subsequent ablation of ovarian A2728 cancer cells. These nanomaterials have shown very interesting properties for applications on theranostic as well as controlled drug-delivery, taking advantage of NIR light absorption and temperature sensitivity (Asadian Birjand et al. 2016).
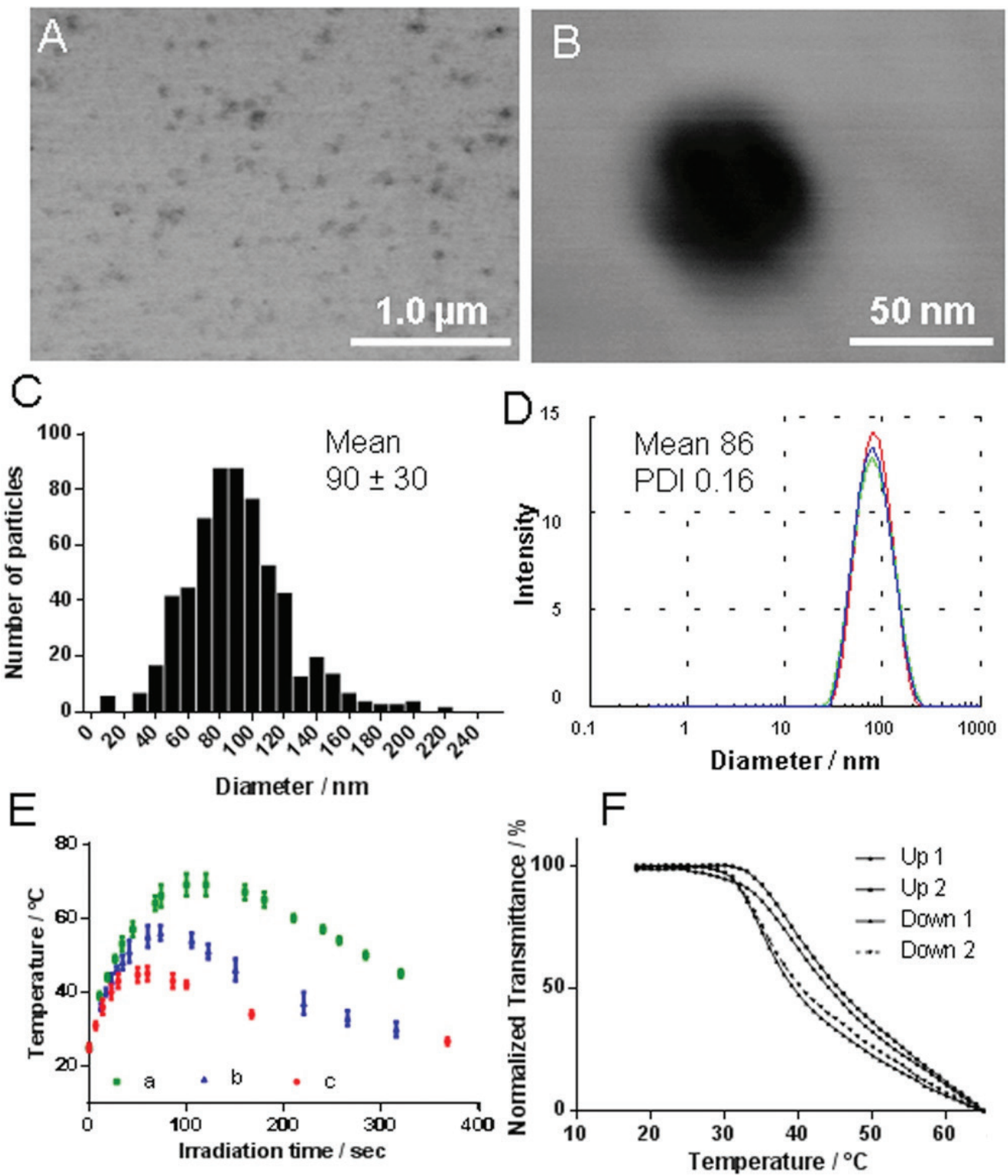

Figure 12. tNG-IR806 characteristics. (A-B) TEM image of uranyl acetate stained tNG-IR806 and zoom. Additional TEM images can be found in Figure S5 in the Supporting Information. (C) Size distribution determined from TEM images by ImageJ software. (D) Hydrodynamic diameter distribution measured by DLS. (E) Heating curves of tNG-IR806 at different aqueous concentrations: a (green) $=12 \mathrm{mg} \mathrm{mL}-1, \mathrm{~b}$ (blue) $=6 \mathrm{mg} \mathrm{mL}-1$, c (red) $=3 \mathrm{mg} \mathrm{mL}-1 \mathrm{upon} \mathrm{NIR}$ laser irradiation at a wavelength of $785 \mathrm{~nm}$ and power density of $0.59 \mathrm{~W} \mathrm{~cm}-2$.Data are expressed as mean $\pm \mathrm{SD}(\mathrm{n}=$ 3). (F) Normalized UV-vis transmittance curves of two heating and cooling cycles in turbidimetry at $450 \mathrm{~nm}$ wavelength. This figure has been reproduced from reference (Asadian Birjand et al. 2016) with permission from John Wiley and Sons and Copyright Clearance Center.

Diverse functional micelles and lipid-based nanoparticles combined with organic dyes are also employed for PTT. For example, Kuang et al. arrive to relevant results referred to as photothermal efficiency by using solid lipid nanoparticles (SLNs) conjugated with c(RGDyK) peptide (cyclo (Arg-Gly-Asp-D-Tyr-Lys)) as a carrier for IR-780 delivery (Kuang et 
al. 2017). cRGD-IR-780 SLNs were produced by using the solvent-diffusion method. The photothermal performance of the synthesized nanoparticles was studied by exposing different concentrations of the nanomaterial under NIR laser ( 808 $\mathrm{nm})$ at $1 \mathrm{~W} \mathrm{~cm}^{-2}$. Whereas the material free of IR-780 in the aqueous solution presented a small increment of temperature, cRGD-IR-780 SLNs showed a clear photothermal effect (an increase of $32^{\circ} \mathrm{C}$, at $50 \mathrm{mg} \mathrm{L}^{-1}$ ). In addition, U87MG cell viability was not affected (no cytotoxicity) and the same cells were killed (viability decrease to $15.1 \%$ ) by application of NIR light. In vivo results using mice reveals a similar photothermal performance as that of in vitro assays, showing a total U87MG tumor regression. These studies were complemented with direct observation of cRGD-IR-780 SLNs, making the material a potential candidate for NIR imaging-guided targeted cancer treatment. By loading the same dye (IR-780) into polymeric micelles, a multi-drug delivery system was proposed for the treatment of colorectal cancer by applying PTT. A series of complete chemical characterization of micelles and testing with HCT-116 and SW-620 cell lines, together with animal models, allows confirming that the novel nanomaterial could be used efficiently for PTT (Shih et al. 2017).

Poly(12-(methacryloyloxy)dodecylphosphorylcholine) (PCB-lipid) micellar nanoparticles encapsulating IR-780 dye were recently reported by Rajendrakumar et al. (Rajendrakumar et al. 2018). The synthesis consists on the additionfragmentation chain transfer (RAFT) polymerization and self-assembly procedure. The material was developed for the treatment of cervical tumor using PTT combined with NIR-based fluorescence imaging. The photothermal effect of the nanoparticles has shown a high-temperature change $\left(\sim 30^{\circ} \mathrm{C}\right)$ under NIR light $\left(808 \mathrm{~nm}, 2 \mathrm{~W} \mathrm{~cm}{ }^{-2}\right)$ for only 5 minutes of irradiation. Besides, good viability of PCB-lipid-IR-780 nanoparticles was observed in cytotoxic studies employing TC-1 cells. A concentration-dependent effect was found in the cell viability reduction when the nanomaterial was exposed upon NIR laser irradiation. In vivo studies in animal tumor models revealed a significant reduction of the tumor volume by PTT mechanisms, demonstrating excellent heat conversion.

Other nanocomposites based on less common materials employing NIR dyes were developed recently. Ferritin nanocages were proposed as a theranostic platform based on new cyanine green (IR-820). The resulting material (spherical particles of c.a. $20 \mathrm{~nm}$ ) have shown NIR absorption, which is of interest for PAI/fluorescence multimodal imaging-guided PTT. In this case, in vitro photothermal assays were carried out on 4T1 cells, showing a high photothermal conversion efficiency after 5 minutes of NIR irradiation $(808 \mathrm{~nm})$ at low powers $\left(0.1,0.5\right.$, and $\left.1 \mathrm{~W} \mathrm{~cm}^{-2}\right)$. Taking into account the presence of NIR fluorescence and PAI effects, the in vivo multimodal imaging-guided PTT was evaluated on mice with subcutaneous 4T1 tumors injected. The changes on tumor temperatures were registered at different laser powers, resulting in $23.8^{\circ} \mathrm{C}\left(0.5 \mathrm{~W} \mathrm{~cm}^{-2}\right)$ and $42.2^{\circ} \mathrm{C}\left(1 \mathrm{~W} \mathrm{~cm}^{-2}\right)$ with $100 \%$ of tumor elimination (Huang et al. 2014). Another example shows how it is possible to employ black phosphorus nanosheets (BPs) to build a novel photothermal agent. The material is similar to graphene nanosheets, but it is constituted by phosphorous 2D layers. The aromatic amine group of Nile Blue dye is diazotized, and the aromatic cation reacted with BPs, obtaining an attached dye NB@BPs. The nanosheets show good optical performance, stability, and high NIR fluorescence. Additionally, the nanomaterials show good biocompatibility and no cytotoxicity. Taking advantage of these properties, the PTT effect was evaluated. The exposition of MCF7 cancer cells to NB@BPs at low concentration (50 ppm) and illumination with NIR light (10 minutes) caused a $90 \%$ decrease in cell viability. Furthermore, the in vivo experiments combining fluorescence imaging and PTT revealed successful tumor ablation by the effect of temperature increasing $\left(\sim 23^{\circ} \mathrm{C}\right)$, demonstrating the potential ability of NB@ BPs to be applied on nanomedicine (Zhao et al. 2017).

\section{Conclusions and future perspectives}

In summary, we have presented a review of the different nanoparticles that are currently being studied in PTT. The main objective of these photothermal agents is to be highly efficient and selective for thermal therapies for diverse diseases, mainly cancer and bacterial infections. It has been shown that many nanoparticles (metallic nanoparticles, conductive polymeric nanoparticles, carbon nanotubes, graphene-based nanoparticles, etc.) can be used as photothermal agents, since they present large light-to-heat conversion efficiencies. Among them, carbon-based and conjugated polymers show good PTT properties as well as good biodegradability.

However, there are still some issues to be solved before translating this platform to the clinic. For example, in order to get the deepest tissue penetration, it is necessary to find the best wavelengths within the biological windows. Some nanomaterials (e.g. PANI) show a clear $\mathrm{pH}$ effect which decreases NIR absorption in the cytoplasm (neutral pH). However, such property could be altered by chemical functionalization, such as self-doping. Moreover, the combination of conjugated polymers with NIR dyes could also increase photothermal efficiency.

On the other hand, in the case of metallic nanoparticles, additional effects such as localized plasmon have been shown to affect catalysis or even liquid boiling. It is likely that such phenomena have effects on cell or bacteria induced death, but it has not been investigated closely. 
As it can be seen in the work reviewed here, the heat production due to NIR light absorption is measured at the macroscopic level as an increase in the temperature of the irradiated zone. However, it is likely that nanomaterial temperature could reach values higher than the mean value of the irradiated zone. Besides, such large temperature changes could be responsible for cell death.

However, there is a lack of real-time and/or local temperature monitoring systems. The best candidate, temperature-dependent fluorescence, does not work, up to now, in the NIR range. On the other hand, thermosensitive polymers have been used to detect local temperature gradients at the nanometric level, but signal detection is somewhat slow.

Hereafter, the development of multifunctional nanoplatforms, which include heating, tracking, and sensing all in one, is expected. This could involve a combination of more than one kind of nanomaterials. The very nature of nanoagents allows combining different materials without undue interactions such as the ones that occur in molecules. We think this will be the main research focus in the field during the next years.

\section{Acknowledgments}

Cuello and Bongiovanni Abel thank CONICET for a graduate fellowship. Molina, Yslas and Barbero are permanent research fellows of CONICET. We thank Juan Manuel Andrea for the graphical abstract design.

\section{References}

[1] G. Y. Ahn. Polyaniline-grafted nanodiamonds for efficient photothermal tumor therapy. Colloids and Surfaces B: Biointerfaces. 180: 273-280, 2019.

[2] D. I. Andersson and D. Hughes. Antibiotic resistance and its cost: is it possible to reverse resistance? Nature Reviews Microbiology. 8: 260, 2010.

[3] M. Asadian Birjand. Near infrared dye conjugated nanogels for combined photodynamic and photothermal therapies. Macromolecular bioscience. 16(10): 1432-1441, 2016.

[4] C. Backes and A. Hirsch. Noncovalent Functionalization of Carbon Nanotubes. In Chemistry of Nanocarbons. John Wiley \& Sons Ltd, United Kingdom, 2010.

[5] E. Birben. Oxidative stress and antioxidant defense. The World Allergy Organization journal. 5(1): 9-19, 2012.

[6] E. Boisselier and D. Astruc. Gold nanoparticles in nanomedicine: preparations, imaging, diagnostics, therapies and toxicity. Chemical Society Reviews. 38(6): 1759-1782, 2009.

[7] S. Bongiovanni Abel. Photothermal lysis of Pseudomonas aeruginosa by polyaniline nanoparticles under near infrared irradiation. Biomedical Physics \& Engineering Express. 4(4): 045037, 2018.

[8] S. Bongiovanni Abel. Smart polyaniline nanoparticles with thermal and photothermal sensitivity. Nanotechnology. 25(49): 495602, 2014.

[9] S. Bongiovanni Abel. Synthesis of polyaniline (PANI) and functionalized polyaniline (F-PANI) nanoparticles with controlled size by solvent displacement method. Application in fluorescence detection and bacteria killing by photothermal effect. Nanotechnology. 29(12): 125604, 2018.

[10] T. Cantu. Conductive polymer-based nanoparticles for laser-mediated photothermal ablation of cancer: synthesis, characterization, and in vitro evaluation. International journal of nanomedicine. 12: 615-632, 2017.

[11] Y. Cao. Near-infrared conjugated polymers for photoacoustic imaging-guided photothermal/chemo combination therapy. Journal of Materials Chemistry B. 5(27): 5479-5487, 2017.

[12] J. Chen. Nanomaterials as photothermal therapeutic agents. Progress in materials science. 2018.

[13] L. Cheng. Functional Nanomaterials for Phototherapies of Cancer. Chemical Reviews. 114(21): 10869-10939, 2014.

[14] L. Cheng. Organic Stealth Nanoparticles for Highly Effective in Vivo Near-Infrared Photothermal Therapy of Cancer. 
ACS Nano. 6(6): 5605-5613, 2012.

[15] P. Fan. GO@Polyaniline Nanorod Array Hierarchical Structure: A Photothermal Agent with High Photothermal Conversion Efficiency for Fast Near-Infrared Responsive Hydrogels. Industrial \& Engineering Chemistry Research. 58(9): 3893-3901, 2019.

[16] Y. Feng. Reduced Graphene Oxide Functionalized with Gold Nanostar Nanocomposites for Synergistically Killing Bacteria through Intrinsic Antimicrobial Activity and Photothermal Ablation. ACS Applied Bio Materials. 2(2): 747$756,2019$.

[17] D. He. Combined photothermal and antibiotic therapy for bacterial infection via acidity-sensitive nanocarriers with enhanced antimicrobial performance. 12: 415-429, 2018.

[18] L. R. Hirsch. Nanoshell-mediated near-infrared thermal therapy of tumors under magnetic resonance guidance. Proceedings of the National Academy of Sciences. 100(23): 13549, 2003.

[19] G. Housman. Drug resistance in cancer: an overview. Cancers (Basel). 6(3): 1769-1792, 2014.

[20] P. Huang. Dye-loaded ferritin nanocages for multimodal imaging and photothermal therapy. Advanced materials (Deerfield Beach, Fla.). 26(37): 6401-6408, 2014.

[21] W.-C. Huang. Functional gold nanoparticles as photothermal agents for selective-killing of pathogenic bacteria. Nanomedicine. 2(6): 777-787, 2007.

[22] X. Huang. Cancer Cell Imaging and Photothermal Therapy in the Near-Infrared Region by Using Gold Nanorods. Journal of the American Chemical Society. 128(6): 2115-2120, 2006.

[23] L. Ibarra. Near-infrared mediated tumor destruction by photothermal effect of PANI-Np in vivo. Laser Physics. 23(6): 066004, 2013.

[24] S. Iijima. Helical microtubules of graphitic carbon. Nature. 354(6348): 56-58, 1991.

[25] P. K. Jain. Au nanoparticles target cancer. Nano Today. 2(1): 18-29, 2007.

[26] P. K. Jain. Calculated Absorption and Scattering Properties of Gold Nanoparticles of Different Size, Shape, and Composition: Applications in Biological Imaging and Biomedicine. The Journal of Physical Chemistry B. 110(14): 72387248, 2006.

[27] D. Jaque. Nanoparticles for photothermal therapies. Nanoscale. 6(16): 9494-9530, 2014.

[28] X. Jia. Versatile graphene-based photothermal nanocomposites for effectively capturing and killing bacteria, and for destroying bacterial biofilms. Journal of Materials Chemistry B. 5(13): 2459-2467, 2017.

[29] H. S. Jung. Organic molecule-based photothermal agents: an expanding photothermal therapy universe. Chemical Society Reviews. 47(7): 2280-2297, 2018.

[30] N. W. S. Kam. Carbon nanotubes as multifunctional biological transporters and near-infrared agents for selective cancer cell destruction. Proceedings of the National Academy of Sciences of the United States of America. 102(33): 11600-11605, 2005.

[31] Y. Ko. Antibacterial poly (3,4-ethylenedioxythiophene):poly(styrene-sulfonate)/agarose nanocomposite hydrogels with thermo-processability and self-healing. Carbohydrate Polymers. 203:26-34, 2019.

[32] C. Korupalli. Acidity-triggered charge-convertible nanoparticles that can cause bacterium-specific aggregation in situ to enhance photothermal ablation of focal infection. Biomaterials. 116:1-9, 2017.

[33] Y. Kuang. Hydrophobic IR-780 dye encapsulated in cRGD-conjugated solid lipid nanoparticles for NIR imaging-guided photothermal therapy. ACS Applied Materials \& Interfaces. 9(14): 12217-12226, 2017.

[34] G. Kucsko. Nanometre-scale thermometry in a living cell. Nature. 500:54, 2013.

[35] S. Link and M. A. El-Sayed. Simulation of the Optical Absorption Spectra of Gold Nanorods as a Function of Their Aspect Ratio and the Effect of the Medium Dielectric Constant. The Journal of Physical Chemistry B. 109(20): 1053110532, 2005. 
[36] S. Link and M. A. El-Sayed. Size and Temperature Dependence of the Plasmon Absorption of Colloidal Gold Nanoparticles. The Journal of Physical Chemistry B. 103(21): 4212-4217, 1999.

[37] X. Liu. Functionalized poly(pyrrole-3-carboxylic acid) nanoneedles for dual-imaging guided PDT/PTT combination therapy. Biomaterials. 167: 177-190, 2018.

[38] K. P. Loh. Graphene oxide as a chemically tunable platform for optical applications. Nature Chemistry. 2: $1015,2010$.

[39] A. Lombardi. Surface Plasmon Resonance Properties of Single Elongated Nano-objects: Gold Nanobipyramids and Nanorods. Langmuir. 28(24): 9027-9033, 2012.

[40] C. Loo. Nanoshell-Enabled Photonics-Based Imaging and Therapy of Cancer. 3(1): 33-40, 2004.

[41] P. A. Mackowiak. Direct Effects of Hyperthermia on Pathogenic Microorganisms: Teleologic Implications with Regard to Fever. Clinical Infectious Diseases. 3(3): 508-520, 1981.

[42] D. G. Meeker. Synergistic Photothermal and Antibiotic Killing of Biofilm-Associated Staphylococcus aureus Using Targeted Antibiotic-Loaded Gold Nanoconstructs. ACS Infectious Diseases. 2(4): 241-250, 2016.

[43] J. U. Menon. Nanomaterials for photo-based diagnostic and therapeutic applications. Theranostics. 3(3): $152,2013$.

[44] N. J. Millenbaugh. Photothermal killing of Staphylococcus aureus using antibody-targeted gold nanoparticles. International journal of nanomedicine. 10: 1953-1960, 2015.

[45] M. Molina. Polymeric near-infrared absorbing dendritic nanogels for efficient in vivo photothermal cancer therapy. Nanoscale. 8(11): 5852-5856, 2016.

[46] B. Nikoobakht and M. A. El-Sayed. Preparation and Growth Mechanism of Gold Nanorods (NRs) Using Seed-Mediated Growth Method. Chemistry of Materials. 15(10): 1957-1962, 2003.

[47] W. H. Organization. ANTIMICROBIAL RESISTANCE. Global Report on surveillance 2014. 2014.

[48] J. Y. Pereyra. Photothermally enhanced bactericidal activity by the combined effect of NIR laser and unmodified graphene oxide against Pseudomonas aeruginosa. Photodiagnosis and Photodynamic Therapy. 24: 36-43, 2018.

[49] F. Pierini. Polymer-based nanomaterials for photothermal therapy: FROM light-responsive to multifunctional nanoplatforms for synergistically combined technologies. Biomacromolecules. 19(11): 4147-4167, 2018.

[50] S. Rajendrakumar. A lipophilic ir-780 dye-encapsulated zwitterionic polymer-lipid micellar nanoparticle for enhanced photothermal therapy and nir-based fluorescence imaging in a cervical tumor mouse model. International journal of molecular sciences. 19(4): 1189, 2018.

[51] J. T. Robinson. Ultrasmall Reduced Graphene Oxide with High Near-Infrared Absorbance for Photothermal Therapy. Journal of the American Chemical Society. 133(17): 6825-6831, 2011.

[52] M. Shahnawaz Khan. Near infrared (NIR) laser mediated surface activation of graphene oxide nanoflakes for efficient antibacterial, antifungal and wound healing treatment. Colloids and Surfaces B: Biointerfaces. 127: 281-291, 2015.

[53] Y.-H. Shih. EGFR-targeted micelles containing near-infrared dye for enhanced photothermal therapy in colorectal cancer. Journal of Controlled Release. 258: 196-207, 2017.

[54] S. Singh. Understanding the Mechanism of Bacterial Biofilms Resistance to Antimicrobial Agents. The open microbiology journal. 11: 53-62, 2017.

[55] Z. Sobhani. Photothermal therapy of melanoma tumor using multiwalled carbon nanotubes. International journal of nanomedicine. 12: 4509-4517, 2017.

[56] J. Song. Sequential Drug Release and Enhanced Photothermal and Photoacoustic Effect of Hybrid Reduced Graphene Oxide-Loaded Ultrasmall Gold Nanorod Vesicles for Cancer Therapy. ACS Nano. 9(9): 9199-9209, 2015.

[57] J. Stejskal and I. Sapurina. Polyaniline: Thin films and colloidal dispersions (IUPAC Technical Report). Pure and Applied Chemistry. 77(5): 815-826, 2005. 
[58] T. Sun. Second Near-Infrared Conjugated Polymer Nanoparticles for Photoacoustic Imaging and Photothermal Therapy. ACS Applied Materials \& Interfaces. 10(9): 7919-7926, 2018.

[59] L. Tan. Rapid Biofilm Eradication on Bone Implants Using Red Phosphorus and Near-Infrared Light. Advanced Materials. 30(31): 1801808, 2018.

[60] V. S. Thakare. Carbon nanotubes in cancer theragnosis. Nanomedicine. 5(8): 1277-1301, 2010.

[61] S. Thota and D. C. Crans. Metal nanoparticles: synthesis and applications in pharmaceutical sciences. John Wiley \& Sons, Wenheim, Germany, 2018.

[62] L. Tong. Gold nanorods as contrast agents for biological imaging: optical properties, surface conjugation and photothermal effects. Photochemistry and photobiology. 85(1): 21-32, 2009.

[63] T. Tsuchido. Destruction of the outer membrane permeability barrier of Escherichia coli by heat treatment. Applied and environmental microbiology. 50(2): 298-303, 1985.

[64] J. Wang and J. Qiu. A review of organic nanomaterials in photothermal cancer therapy. 2016.

[65] J. Wang and J. Qiu. A review of organic nanomaterials in photothermal cancer therapy. Cancer Research Frontiers. 2(1): $67-84,2016$.

[66] J. Wang. Controllable Synthesis of Gold Nanorod/Conducting Polymer Core/Shell Hybrids Toward in Vitro and in Vivo near-Infrared Photothermal Therapy. ACS Applied Materials \& Interfaces. 10(15): 12323-12330, 2018.

[67] X.-H. Wang. Indocyanine green-platinum porphyrins integrated conjugated polymer hybrid nanoparticles for near-infrared-triggered photothermal and two-photon photodynamic therapy. Journal of Materials Chemistry B. 5(9): 1856$1862,2017$.

[68] X. Wang. Enhanced photothermal therapy of biomimetic polypyrrole nanoparticles through improving blood flow perfusion. Biomaterials. 143: 130-141, 2017.

[69] X. Wang. Fabrication of Ultralong and Electrically Uniform Single-Walled Carbon Nanotubes on Clean Substrates. Nano Letters. 9(9): 3137-3141, 2009.

[70] Y. Wang. Comparison Study of Gold Nanohexapods, Nanorods, and Nanocages for Photothermal Cancer Treatment. ACS Nano. 7(3): 2068-2077, 2013.

[71] R. Weissleder. A clearer vision for in vivo imaging. Nature Biotechnology. 19: 316, 2001.

[72] M.-C. Wu. Graphene-Based Photothermal Agent for Rapid and Effective Killing of Bacteria. ACS Nano. 7(2): 1281$1290,2013$.

[73] J.-W. Xu. Nanomaterials with a photothermal effect for antibacterial activities: an overview. Nanoscale. 2019.

[74] X. Xu. Controlled-temperature photothermal and oxidative bacteria killing and acceleration of wound healing by polydopamine-assisted Au-hydroxyapatite nanorods. Acta Biomater. 77: 352-364, 2018.

[75] K. Yang. Graphene in Mice: Ultrahigh In Vivo Tumor Uptake and Efficient Photothermal Therapy. Nano Letters. 10(9): 3318-3323, 2010.

[76] S. Yang. Multifunctional Bi@PPy-PEG Core-Shell Nanohybrids for Dual-Modal Imaging and Photothermal Therapy. ACS Applied Materials \& Interfaces. 10(2): 1605-1615, 2018.

[77] Y. Yang. Nonchemotherapic and Robust Dual-Responsive Nanoagents with On-Demand Bacterial Trapping, Ablation, and Release for Efficient Wound Disinfection. Advanced Functional Materials. 28(21): 1705708, 2018.

[78] E. I. Yslas. Polyaniline nanoparticles for near-infrared photothermal destruction of cancer cells. Journal of Nanoparticle Research. 17(10): 389, 2015.

[79] C. Yue. IR-780 dye loaded tumor targeting theranostic nanoparticles for NIR imaging and photothermal therapy. Biomaterials. 34(28): 6853-6861, 2013. 
[80] Z. Zha. Polypyrrole Hollow Microspheres as Echogenic Photothermal Agent for Ultrasound Imaging Guided Tumor Ablation. Scientific Reports. 3: 2360, 2013.

[81] Z. Zha. Uniform Polypyrrole Nanoparticles with High Photothermal Conversion Efficiency for Photothermal Ablation of Cancer Cells. Advanced Materials. 25(5): 777-782, 2013.

[82] B. Zhang. Fibrin-targeting peptide CREKA-conjugated multi-walled carbon nanotubes for self-amplified photothermal therapy of tumor. Biomaterials. 79: 46-55, 2016.

[83] Y. Zhao. Stable and Multifunctional Dye-Modified Black Phosphorus Nanosheets for Near-Infrared Imaging-Guided Photothermal Therapy. Chemistry of Materials. 29(17): 7131-7139, 2017.

[84] Z. Zhao. Bacteria-Activated Theranostic Nanoprobes against Methicillin-Resistant Staphylococcus aureus Infection. ACS Nano. 11(5): 4428-4438, 2017.

[85] V. P. Zharov. Photothermal Nanotherapeutics and Nanodiagnostics for Selective Killing of Bacteria Targeted with Gold Nanoparticles. Biophysical Journal. 90(2): 619-627, 2006.

[86] Y. Zhou. Photoresponsive Drug/Gene Delivery Systems. Biomacromolecules. 19(6): 1840-1857, 2018.

\section{Bios}

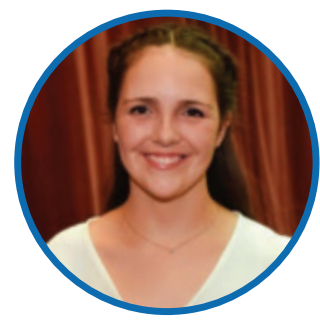

\section{Emma Antonia Cuello}

Emma Antonia Cuello obtained her B. Sc. in Chemistry from the National University of Río Cuarto (Argentina) in 2018. For her bachelor thesis she worked on the synthesis and characterization of smart materials for bacterial growth inhibition under supervision of Prof. Diego Acevedo. She is currently a second year PhD student under supervision of Prof. Cesar Barbero (National University of Río Cuarto). Her research program is focused on the development of actuators based on gel matrix nanocomposites for different technological applications.

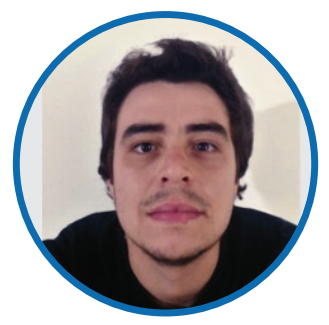

Silvestre Bongiovanni Abel

Silvestre Bongiovanni Abel obtained both his B.Sc. (2013) and Ph.D. in Chemistry (2018) from the National University of Rio Cuarto (Argentina). He was a CONICET Postdoctoral fellow at INTEMA (CONICET-UNMdP) working in electrohydrodynamic techniques for the obtention of polymeric structures with controlled 3D architecture, under the supervision of Prof. Gustavo Abraham. In 2020, he joined the Responsive Polymer Therapeutics Group (POLYMAT, Basque Center for Macromolecular Design and Engineering, Spain) as a Postdoctoral Researcher. Recently, he was named Assistant Researcher of CONICET. His research interests include the synthesis and development of nanomaterials based on conducting and smart polymers for biomedical applications.

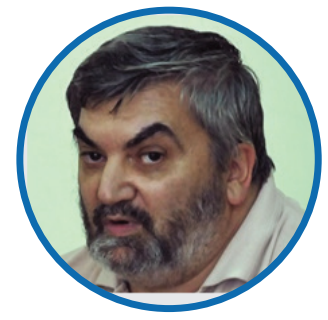

\section{César Barbero}

Dr. César Barbero received his B.Sc (1984, s.c.l.) and Ph.D. (1988, hons) in Chemistry from the National University of Rio Cuarto (UNRC, Argentina). After a postdoctoral stay (1988-1994) at Paul Scherrer Intitute (Switzerland), he returned to the UNRC to become an Assistant Professor. At present, he is Full Professor in Chemistry and Superior Researcher of the National Science Council (CONICET), Argentina. He is the Director of the Research Institute on Energy Technologies and Advanced Material (UNRC-CONICET). He has advised $13 \mathrm{Ph} . \mathrm{D}$. thesis, published over 180 manuscripts $(\mathrm{h}=34)$ and holds 6 patents. He received the Tajima Prize of the International Society of Electrochemistry (1997).

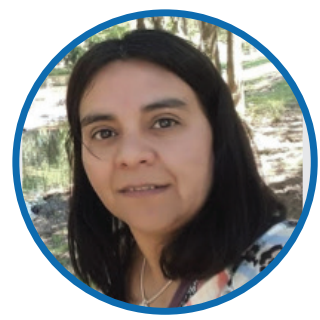

\section{Inés Yslas}

Dr. E. Inés Yslas received a degree in Microbiology from the National University of Río Cuarto (UNRC, Argentina) in 2000. In 2002, she obtained a CONICET Fellowship to undertake her PhD studies working on photodynamic therapy in cancer. From 2006 to 2008 she was a CONICET postdoctoral fellow, working on photothermal therapy in cancer. Since 2008, she is a scientific researcher of CONICET at the UNRC working in photothermal therapy in bacterial infections and antibacterial surfaces. 


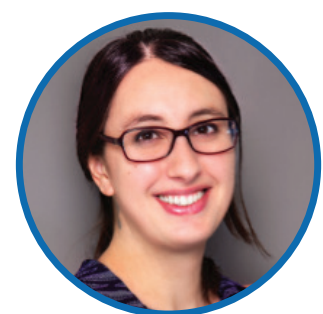

\section{María Molina}

Dr. María Molina received her BSc

(Hons) in Chemistry from the Universidad Nacional de Rio Cuarto (UNRC, Argentina) in 2007. Also in 2007, she obtained a CONICET Fellowship to undertake her $\mathrm{PhD}$ studies (2011) in Prof. Cesar Barbero's group (UNRC) working on the preparation of smart hydrogels as drug delivery systems. From 2012 to 2015, she was an Alexander von Humboldt postdoctoral fellow at Freie Universität Berlin, under the supervision of Prof. M. Calderón and Prof. R. Haag. Since 2017, she is a research scientist of CONICET at the UNRC. She received the International Rising Talents Awards from Loreal-UNESCO For Women in Science program in 2019. 